\title{
Star formation in the Cepheus Flare region: implications from morphology and infrared properties of optically selected clouds ${ }^{\star} \star \star$
}

\author{
Z. T. Kiss ${ }^{1,2}$, L. V. Tóth ${ }^{2,3,5}$, O. Krause ${ }^{4}$, M. Kun ${ }^{5}$, and M. Stickel ${ }^{4}$ \\ 1 Baja Astronomical Observatory, PO Box 766, 6500 Baja, Hungary \\ e-mail: kissz@alcyone.bajaobs.hu \\ 2 Eötvös Loránd University, Department of Astronomy, PO Box 32, 1518 Budapest, Hungary \\ 3 Observatory, Tähtitorninmäki, 00014 Helsinki University, Finland \\ 4 Max Planck Institut für Astronomie, Königstuhl 17, 69117 Heidelberg, Germany \\ 5 Konkoly Observatory of the Hungarian Academy of Sciences, PO Box 67, 1525 Budapest, Hungary
}

Received 13 April 2005 / Accepted 21 February 2006

\section{ABSTRACT}

\begin{abstract}
Aims. A complex study of visual and infrared properties of ISM and an examination of cloud morphology was carried out using USNO, 2MASS, DIRBE, IRAS, and ISO data of an extended region in Cepheus to explore the conditions of cloud and star formation. Methods. We mapped the distribution of visual extinction in a 256 square degree area of Cepheus. We identified clouds and described their morphology quantitatively to find features such as globular and head-tail shape. We also characterized the region using infrared data and combined the results with those obtained from visual data.

Results. Eight cloud complexes and four voids were identified and 208 dark clouds were localised, 86 of which had not been previously catalogued. The observed distribution of cloud axis ratios corresponds to near-prolate ellipsoidal clouds with random alignment. The cloud mass spectrum derived is $\mathrm{d} N_{\text {cloud }} / \mathrm{d} M_{\text {cloud }}=6.1 \times 10^{2}\left(M_{\text {cloud }} / M_{\odot}\right)^{-1.70} M_{\odot}^{-1}$. We pointed out a linear relationship between FIR colour temperature and galactic latitude at intermediate galactic latitudes $\left(3^{\circ}<b<18^{\circ}\right)$ and a similar relationship for minimum cloud temperatures. The observed FIR colours of dark clouds were found to agree well with model calculations. We showed the signs of connection between the far-IR loop GIRL G109+11 and the active triggered star formation of adjacent cloud complexes. Relationships between the star-forming efficiency, peak extinction, and cloud mass were recognized and described with empirical formulae. YSOs were found in 7 of the 11 clouds with extinction above 4 mag. These 11 clouds represent $21 \%$ of the total cloud mass and contain $52 \%$ of all the YSO candidates. High star-forming efficiencies, $0.52 \%$ and $0.56 \%$, were found in globular and head-tail clouds, respectively, relative to the mean efficiency for the clouds in the region $(0.16 \%)$.

Conclusions. Cloud mass and peak extinction were found to be the most important factors of star-forming efficiency besides cloud morphology. The estimated intrinsic axis-ratio distribution is consistent with cloud formation from large-scale external forcing.
\end{abstract}

Key words. ISM: clouds - dust, extinction - ISM: structure - stars: formation - Galaxy: solar neighbourhood - infrared: ISM

\section{Introduction}

We present a statistical study investigating the ISM structure and star-forming activity in Cepheus, based on an analysis of the distribution of extinction and radiation by dust. The motivation for studying cloud morphology is to understand the physical processes that create clouds both as transient features and as star-forming sites. Since most clouds are bound by external pressure, their shape is mainly caused by external effects (Ward-Thompson 2002). A study of cloud morphology will provide us with indirect information on these external effects.

Assuming that the ratio of dust extinction (at a fixed wavelength) to gas column density is constant, one can use extinction

$\star$ Based on observations with ISO, an ESA project with instruments funded by ESA Member States (in particular the PI countries: France, Germany, the Netherlands, and the UK) and with the participation of ISAS and NASA.

$\star \star$ Tables A.1 and A.2 are only available in electronic form at the CDS via anonymous ftp to

cdsarc.u-strasbg.fr $(130.79 .128 .5)$ or via

http://cdsweb.u-strasbg.fr/cgi-bin/qcat?]/A+A/453/923 to map the mass distribution in clouds. During the last decades $\mathrm{CO}$ lines were proven to be effective probes of cloud morphology, and ${ }^{13} \mathrm{CO}(1-0)$ intensity was found to be in linear correlation with $A_{\mathrm{V}}$ in the $1.5 \mathrm{mag}<A_{\mathrm{V}}<5 \mathrm{mag}$ range. As a result of surveys of ${ }^{13} \mathrm{CO}$ emission by Yonekura et al. (1997), for example, the mass spectrum of interstellar clouds was derived for several regions. Cloud shapes were discussed by Jones \& Basu (2002), among others, and the implications of the observed cloud-mass spectrum were given, for instance, by Evans (1999). It is worth investigating whether an unbiased large area survey of extinction gives the same results.

A complex comparative study of cloud morphology, FIR parameters, and associated IR and $\mathrm{H} \alpha$ point sources may reveal the relation of intrinsic morphology to further structuring and star formation. A proper region for such a study must lie outside the immediate vicinity of $\mathrm{OB}$ associations, but have a high enough number and variety of clouds for a statistical approach. In addition, a survey of FIR emission beyond $100 \mu \mathrm{m}$ should be available for a reliable study of the physical parameters of cold $\left(T_{\mathrm{d}}<18 \mathrm{~K}\right)$ clouds with an appropriate resolution $(F W H M<$ expected cloud size). The Cepheus Flare region (Hubble 1934; 
Lebrun 1986) fulfils these criteria. It is known as a site of low- and intermediate-mass star formation with cold and dense clouds, as derived from IRAS data (see e.g. Kun 1998). The distribution of ${ }^{13} \mathrm{CO}$ was mapped there by Yonekura et al. (1997) with an undersampled survey ( $8^{\prime}$ grid, $2^{\prime}$. 7 beam $)$ in a $30^{\circ} \times 30^{\circ}$ field in Cepheus and Cassiopeia, and 188 clouds were found with a total mass of $10^{5} M_{\odot}$. Due to mission planning constraints, the Upper-Cepheus was observed by ISO with a very high sky-coverage rate in the Serendipity Survey mode (Bogun et al. 1996) of the ISOPHOT C200 camera (Lemke et al. 1998), which makes the region unique.

Kun (1998) presented a detailed investigation of cloud-layer distances and concluded that the Cepheus Flare giant molecular cloud (Lebrun 1986) lies at $300 \pm 30 \mathrm{pc}$ and that there are further ISM layers at $450 \mathrm{pc}$ and $200 \mathrm{pc}$ distances. The catalogue of Galactic Infrared Loops created by Kiss et al. (2004) lists 7 large $100 \mu \mathrm{m}$ features in this region. Some of these, e.g. GIRL G109+11, are seen in $\mathrm{HI} 21 \mathrm{~cm}$ (Burton \& Hartmann 1994) as well. An investigation of possible cloud reshaping and triggering phenomena was carried out by Tóth \& Horváth (1996); however, it was limited to the analysis of the largest clouds of the Yonekura et al. (1997) survey.

The classical way to locate nearby interstellar clouds is to map extinction using star counts (Dickman 1978). This method was recently revised using an adaptive grid (e.g. Cambrésy 1999a). Extinction maps of individual subfields of the Cepheus region were given by Harjunpää et al. (1991) for L 1155/L 1157 and by Balázs et al. (2004) for L 1251, and an all-sky map of extinction was presented by Cambrésy (1999b), although without identifying substructures.

We report our results on the structure of ISM and star formation in the Cepheus Flare region based on new extinction and ISOSS $170 \mu \mathrm{m}$ data. We located clouds, cloud complexes, and voids using the revised method of extinction mapping based on visual data. The differences obtained using the more recent method of extinction mapping based on near-infrared stellar colours utilising 2MASS data are also discussed. We introduce a quantitative morphological description of dark clouds, and we also derive quantities to describe their infrared characteristics. We calculate the global trends and large-scale variations in the region, and, if possible, remove them to avoid their bias in the characterisation of individual clouds. We describe the region on larger scales than those of individual clouds, and we define and describe subregions that seem to be distinct from each other.

Our goal is to find parameters, or combination of parameters, including visual and IR characteristics and morphology, that can distinguish star-forming clouds from non-star-forming ones. We pay attention to clouds with special morphologies that can be important with respect to star formation, i.e. globular and headtail clouds.

\section{Input data and data processing}

\subsection{Extinction map}

We constructed the extinction map of the region using two different methods. A large, 256 square degree area mosaic in a $18^{\circ} \times 18^{\circ}$ field of Cepheus was analysed with a field centre of $\mathrm{RA}(\mathrm{J} 2000)=21^{\mathrm{h}} 40^{\mathrm{m}}, \operatorname{Dec}(\mathrm{J} 2000)=73^{\circ}$. Both optical and NIR-based maps were created on a 1.5 reseau.

Optical star-count-based map: The first map was made based on the revised star-count method in an optical band. The relatively low stellar density, determined by the band used and the limiting magnitude, allows us to trace the extinction reliably only in a limited range. Applying a simple, regular grid method (Dickman 1978), one would be able to give only a lower estimation of extinction for the most opaque regions where stellar density drops below one star per cell. The adaptive grid method (Cambrésy 1999a) avoids this disadvantage by fixing the number of stars instead of the cell size. However, its resolution decreases with the decreasing stellar number density, and therefore the values of extinction obtained for opaque peaks are averages of areas larger than those for the lower density medium. Thus, it still underestimates the actual extinction. Nevertheless, the opticalcount-based method has the advantage of measuring the visual extinction using optical band data; therefore, the conversion to the equivalent extinction $A_{\mathrm{V}}$ is less sensitive to the consideration of the total-to-selective extinction ratio.

The star-count analysis was carried out using the USNO A2.0 catalogue (Monet et al. 1998). The USNO catalogue contains the complete sample of point sources up to $19 \mathrm{mag}$ in $B$ band. An adaptive mesh was applied, as described by Cambrésy (1999a). The extinction at a given position was derived using the relation:

$A_{B}^{\mathrm{USNO}}=\frac{1}{b_{B}} \log \left(\frac{\overline{d_{c l}}}{\overline{d_{c p}}}\right)^{2}$,

where $\overline{d_{c l}}$ is the actual mean separation of the 10 nearest stars around the given position, $\overline{d_{c p}}$ is the modelled mean separation of the 10 nearest stars at the same position without cloud extinction (obtained using the Balázs et al. 2004 implementation of the galaxy model by Wainscoat et al. 1992), and $b_{B}$ is defined by the equation:

$\log \left(\overline{d_{c p}}\right)^{-2}=a_{B}+b_{B} \cdot m_{B}$

where $m_{B}$ is the limiting magnitude. USNO data are missing around the positions of some saturated bright stars. These positions were excluded from mapping. The visual extinction was calculated as

$A_{\mathrm{V}}^{\mathrm{USNO}}=0.76 A_{B}^{\mathrm{USNO}}$

assuming a total-to-selective extinction ratio of $R_{\mathrm{V}}=3.1$. Due to the method used, the nominal error $\sigma_{A_{\mathrm{V}}^{\text {USNO }}}$ does not depend on the local stellar density but only on the reference value $b_{B}$. Since $b_{B}$ mostly depends on the galactic latitude, the error increases from $0.6 \mathrm{mag}$ to $1.0 \mathrm{mag}$ in the latitude range $3^{\circ}<b<$ $27^{\circ}$, and its mean value is $0.8 \mathrm{mag}$. The resolution of the map varies between $2^{\prime}$ and $8^{\prime}$, depending on the local stellar density; its mean value is $3^{\prime}$. In the classical fashion, the optical star-count-based extinctions were used for cloud definition and morphology analysis (see Sect. 3). The extinction map is shown in Fig. 1.

NIR stellar-colour-based map: The second method is based on the measurement of near-infrared colour excesses of background stars. This allows us to make a deeper extinction map with similar or even higher resolution, but this map does not directly trace the distribution of visual extinction. However, since in the last 100 years dark clouds were defined and catalogued by their visual extinction, we derived the distribution of the equivalent $A_{\mathrm{V}}^{2 \mathrm{MASS}}$ from the data of the 2MASS Point Source Catalog (Cutri et al. 2003) according to the relation:

$A_{\mathrm{V}}^{2 \mathrm{MASS}}=b_{J H} E(J-H)+b_{H K} E(H-K)$, 
where $b_{J H}$ and $b_{H K}$ are coefficients that include the conversion factors to equivalent $A_{\mathrm{V}}$ and that weight factors optimized to give minimum variance in extinction (see Lombardi \& Alves 2001). As this method characterises the equivalent optical extinction, $A_{\mathrm{V}}$, from NIR data, it is more sensitive to the variations in the actual value of the total-to-selective extinction ratio, which is assumed to be constant. Using this method and considering only those positions where the obtained value was derived from the data of more than three individual stars, we can reliably trace the extinction up to 8 mag with a resolution of $3^{\prime}$ from the values obtained for individual stars, using weighted means and Gaussian smoothing. Suspected embedded stars selected based on their colour indices were excluded from the mapping. The nominal error of $A_{\mathrm{V}}^{2 \mathrm{MASS}}$ is $\sigma_{A_{\mathrm{V}}^{2 \mathrm{MASS}}}=1.5 \mathrm{mag}$. This map was used only to characterise the peak values of the extinction.

Foreground stars: We made efforts to reduce the effects of foreground stars, but without knowing the distance to each individual star, this can only be done with a statistical approach. To check the contamination by foreground stars, we first made a rough estimation of such an occurrence based on the Wainscoat galaxy model (Wainscoat et al. 1992), and found it to be at most $1 \%$. From this estimation, we calculated its effects on our beam, according to Cambrésy et al. (2002). The given fraction of foreground stars does not cause any effect on the beam for most of the low extinction positions. Even at positions affected by foreground contamination, its effect is smaller than the nominal error of the extinction. The effect becomes comparable to the nominal error above the $2.5 \mathrm{mag}$ extinction level, while higher values are probably underestimated.

We also applied a correction for the values of extinction that were possibly affected by foreground stars. For the positions where the extinction $A_{\mathrm{V}}^{\mathrm{USNO}}$ exceeds $2.5 \mathrm{mag}$, we calculated a corrected value to filter out suspected foreground stars. If any of the stars used for the calculation of the extinction at the given po-

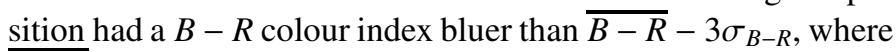
$\overline{B-R}$ and $\sigma_{B-R}$ are the mean and the standard deviation of $B-R$ colour indices of stars used at that position, respectively (i.e. the star is significantly bluer than the mean), we substituted this star with the nearest redder star in the calculation of the corrected value. We checked all the 6005 such positions and found suspected foreground stars at 319 of them, in agreement with the expected frequency according to the galaxy model. The largest difference obtained was $0.7 \mathrm{mag}$, while the mean value was less than $0.1 \mathrm{mag}$. The actual resolution, however, decreased at these positions.

We calculated the NIR-based extinction map using a weighted mean, both with and without sigma clipping. The sigma-clipping method applies iterations to select those extinction values derived from colour excess of individual stars, which are within $3 \sigma$ from the weighted mean (see Lombardi \& Alves 2001). The values of the map, in comparison to the simple mean map, show a scatter of $0.08 \mathrm{mag}$ around the fitted line, which has a slope of 1.00. The sigma-clipped map shows slightly lower extinction values, on average, suggesting that there were more relatively red stars clipped from the sample than relatively blue ones. This may occur because the effect of unresolved, very dense clumps, or stars with extremely red intrinsic colours, are comparable to or stronger than the effect of suspected foreground stars having relatively blue colours, in those positions, where the surface number density of stars is low. That is why the values obtained using this method are reliable only below 8 mag. The extreme deviations caused by sigma clipping are 4.4 mag above and 4.6 mag below the values of the simple weighted mean map. We checked the absolute change in the local mean extinction over various regions on the scale of the expected cloud size and found it to be at most $\pm 0.06 \mathrm{mag}$, which is much smaller than the uncertainties from other known sources. We concluded that the contamination of foreground stars does not significantly affect the extinction values.

\subsection{Cloud definition}

Dark clouds were defined based on the $A_{\mathrm{V}}^{\mathrm{USNO}}$ map by an automatic procedure to allow for the statistical analysis of the morphological, optical, and FIR characteristics of an unbiased cloud sample. We carried out the cloud definition by the following steps:

1) find the highest $A_{\mathrm{V}}^{\mathrm{USNO}}>2.0 \mathrm{mag}$ maximum in the extinction map;

2) find the nearest half-maximum contour;

3) find the cloud boundary knowing that:

a) where the half-maximum contour does not include a formerly defined cloud, it is accepted as the cloud boundary;

b) where the half-maximum contour includes a formerly defined cloud, boundary contour level is increased in 0.1 mag steps till the new peak becomes separate from the formerly defined clouds.

We repeated these steps, searching the mapped area, excluding that covered by formerly found clouds, till new $A_{\mathrm{V}}^{\mathrm{USNO}}>2.0 \mathrm{mag}$ peaks were found. Accordingly, the clouds were detected in decreasing order of their $A_{\mathrm{V}}^{\mathrm{USNO}}$ peak extinction. Clouds reaching the boundary of the map were excluded from the cloud sample.

Cloud boundaries can also be defined with a fixed limiting contour level (e.g. Dobashi et al. 2005). However in this case, the difference in the background levels of isolated clouds and members of cloud complexes may bias the cloud sizes. This may result in a sample containing objects of quite different sizes, from small, isolated globules, to large, highly structured complexes, that is, objects with different levels of hierarchy. We intended to avoid this mixing in hierarchy, however, we will later compare our sample with samples allowing hierarchy (see Sect. 4). We note that both cloud boundary definitions are arbitrary.

\subsection{Cloud morphology analysis}

Parameters were defined to allow a quantitative description of dark cloud morphology. The shapes were approximated with ellipses fitted to the boundaries of the clouds (see above). The centre of the ellipse was placed at the geometrical centre of the cloud (i.e. the simple arithmetic mean of the coordinates of the points inside the boundary), and the axes were defined to cover a region with area equal to that of the cloud and to give the maximum possible value for the total extinction inside the ellipse. We also defined a displacement vector from the geometrical centre to the position of the extinction peak. The morphology parameters were defined as follows:

- $a$ (major axis length) - the major axis of the ellipse defined above;

- $b / a$ (axis ratio) - the axis ratio of the ellipse defined above;

- PA (position angle) - the ccw angle between the direction of the northern galactic pole and the major axis of the ellipse defined above;

- $d p$ (displacement) - the distance between the geometrical centre and the peak; 
- dpa (angle of displacement) - the ccw angle between the major axis and the displacement vector;

- irr (irregularity) $-S_{\text {be }} / S_{\text {cloud }}$, i.e. the ratio of the cloud area inside the boundaries, but outside the ellipse defined $\left(S_{\text {be }}\right)$, to the total area of the cloud $\left(S_{\text {cloud }}\right)$;

- sym (symmetry) - the ratio of the sums of extinction in the two halves of the cloud separated by the minor axis $($ sym $<1)$;

- sep (separateness) - $1-\left(\sum_{\text {ring }} A_{\mathrm{V}}^{\mathrm{USNO}} / \sum_{\mathrm{ell}} A_{\mathrm{V}}^{\mathrm{USNO}}\right)$, where $\sum_{\mathrm{ell}} A_{\mathrm{V}}^{\mathrm{USNO}}$ is the sum of extinction $A_{\mathrm{V}}^{\mathrm{USNO}}$ in the ellipse defined above, and where $\sum_{\text {ring }} A_{\mathrm{V}}^{\mathrm{USNO}}$ is the sum of extinction $A_{\mathrm{V}}^{\mathrm{USNO}}$ in the elliptical ring between the defined ellipse (with axes $a, b$ ) and that enlarged by a factor of 1.41 (with axes $1.41 \times a$ and $1.41 \times b$ ).

These parameters may help us to find some morphological features that we expect exist. Globular clouds, in particular, are expected to be well-separated from the surrounding clouds and to have an axis ratio near 1 , while head-tail-like clouds are expected to be rather elongated and asymmetric along their major axis and to have a relatively large peak displacement in a direction near that of the major axis. Both of them are expected to have relatively low values of irregularity.

\subsection{FIR data}

We used the ISOPHOT Serendipity Survey (Bogun et al. 1996) $170 \mu \mathrm{m}$ and IRAS (Wheelock et al. 1991) ISSA $12 \mu \mathrm{m}, 60 \mu \mathrm{m}$, and $100 \mu \mathrm{m}$ data to characterise the emission of the dust in the region. The ISOSS data were processed as described in Stickel et al. (1998), although details including calibration accuracies are also given by Müller et al (2002). The calibration of the processed ISOSS slews were further optimized, minimising the reproducibility error measured at the slew crossings. Details on the optimization procedure are given in Krause (2003). The absolute photometric accuracy of the resulting $170 \mu \mathrm{m}$ map is $\approx 20 \%$, and the reproducibility error is less than $5 \%$ (rms). A comparison with DIRBE data following Tóth et al. (2000) showed the relation:

$I_{\text {DIRBE }}(170)=1.10 \times I_{\text {ISO }}(170)-3.61$,

a transformation that was applied to put all the FIR data on the same absolute scale. As the DIRBE instrument has not provided data at $170 \mu \mathrm{m}$, these surface brightness values were interpolated from DIRBE 100, 140, and $240 \mu \mathrm{m}$ data, using a modified Planck function and assuming a $\beta=2$ emissivity law.

IRAS surface brightness data were calibrated with the corresponding DIRBE (Hauser et al. 1997) ZSMA data of the studied area. The following correction relations were found and applied:

$$
\begin{aligned}
& I_{\text {DIRBE }}(12)=1.04 \times I_{\text {IRAS }}(12)+0.78, \\
& I_{\text {DIRBE }}(60)=0.78 \times I_{\text {IRAS }}(60)+1.30, \text { and } \\
& I_{\text {DIRBE }}(100)=0.68 \times I_{\text {IRAS }}(100)+2.06,
\end{aligned}
$$

where $I_{\text {DIRBE }}$ and $I_{\text {IRAS }}$ are the DIRBE and IRAS surface brightnesses, respectively.

To avoid a surface brightness contribution by bright point sources, we excluded circular regions with radii of $r=2^{\prime}$ around all point sources listed in the IRAS Point Source Catalogue (IPSC, Beichman et al. 1988) and IRAS Faint Source Catalogue (IFSC, Moshir et al. 1990).

\subsection{Distances to the clouds}

The clouds of our sample are situated in the region that was formerly examined by Kun (1998). Based on Wolf diagrams, Kun found four main components in the region at different distances. We adopted the distance values of the respective components for most of our clouds. We also used distance values given by Racine (1968) for the regions around Cep R1 and Cep R2. For the positions of some clouds, velocity data were presented by Yonekura et al. (1997), Taylor et al. (1987), and Clemens \& Barvainis (1988). We only used these velocity data to decide which of the components actually contains the cloud, if there were clouds at various distance layers. We also used velocity data obtained from our own observations with the KOSMA 3 m telescope (Kiss et al. 2004). In five cases, we found significant amounts of molecular matter in two overlapping components along the line of sight. In one case (G105.1+13.1), we found three distinct velocity components. For the clouds in which more than one velocity component was detected, we assumed that the matter causing optical extinction was situated in the nearest component. The distance values with references are listed in Table A.1. Clouds of different components are plotted in Fig. 1. Adopted distance data were used for mass estimation, using the formula given by Dickman (1978):

$M=(\alpha d)^{2}\left(N_{\mathrm{H}_{2}} / A_{\mathrm{V}}\right) \mu \sum_{\text {cld }} A_{\mathrm{V}}^{\mathrm{USNO}}$,

where $\alpha$ is the reseau size in radians $(=1.5), d$ is the distance to the cloud, $\left(\mathrm{N}_{\mathrm{H}_{2}} / A_{\mathrm{V}}\right)$ is the standard gas-to-extinction ratio $1.25 \times$ $10^{21} \mathrm{~cm}^{-2} \mathrm{mag}^{-1}$ (Jenkins \& Savage 1974), and $\mu$ is the mean particle mass $2.3 \times 10^{-24} \mathrm{~g}$ (Allen 1973).

\subsection{YSO candidates}

We checked the star-forming efficiency of our clouds, considering the sample of candidate young sources of 2MASS PSC (Cutri et al. 2003), IPSC (Beichman et al. 1988), IFSC (Moshir et al. 1990), and $\mathrm{H} \alpha$ emission stars (Kun 1998).

2MASS point sources: Using the new release of the 2MASS PSC (Cutri et al. 2003), we carried out a photometric classification based on $J-H$ vs. $H-K_{\mathrm{s}}$ colour-colour diagrams of sources in our field. We considered sources with quality flags $\mathrm{A}, \mathrm{B}$, or $\mathrm{C}$ and photometric uncertainties of less than 0.1 mag in all three bands. We accepted the sources occupying the locus of classical T Tauri stars (Meyer et al. 1997), i.e. those stars having the whole $1 \sigma$ uncertainty ellipses of their colour indices to the right of the reddened MS in the $J-H$ vs. $H-K_{\mathrm{s}}$ colour-colour diagram, as YSO candidates. As many as 61 such sources were found.

IRAS point sources: YSO candidates as FIR point sources were selected from IPSC and IFSC. The criteria were: $i$ ) definite detection (flux quality of 2 or 3) at either $25 \mu \mathrm{m}$ or both $60 \mu \mathrm{m}$ and $100 \mu \mathrm{m}$, and $i$ ) in the case of $12 \mu \mathrm{m}$ detection, $F_{25}$ should be larger than $F_{12}$, as defined by Beichman et al. (1986) for YSOs.

$\mathrm{H} \alpha$ emission stars: Candidate pre-main-sequence stars have been taken from Kun (1998).

Galaxy removal: Galaxies were removed from the combined sample using the HYPERLEDA (Paturel et al. 2003) catalogue, 


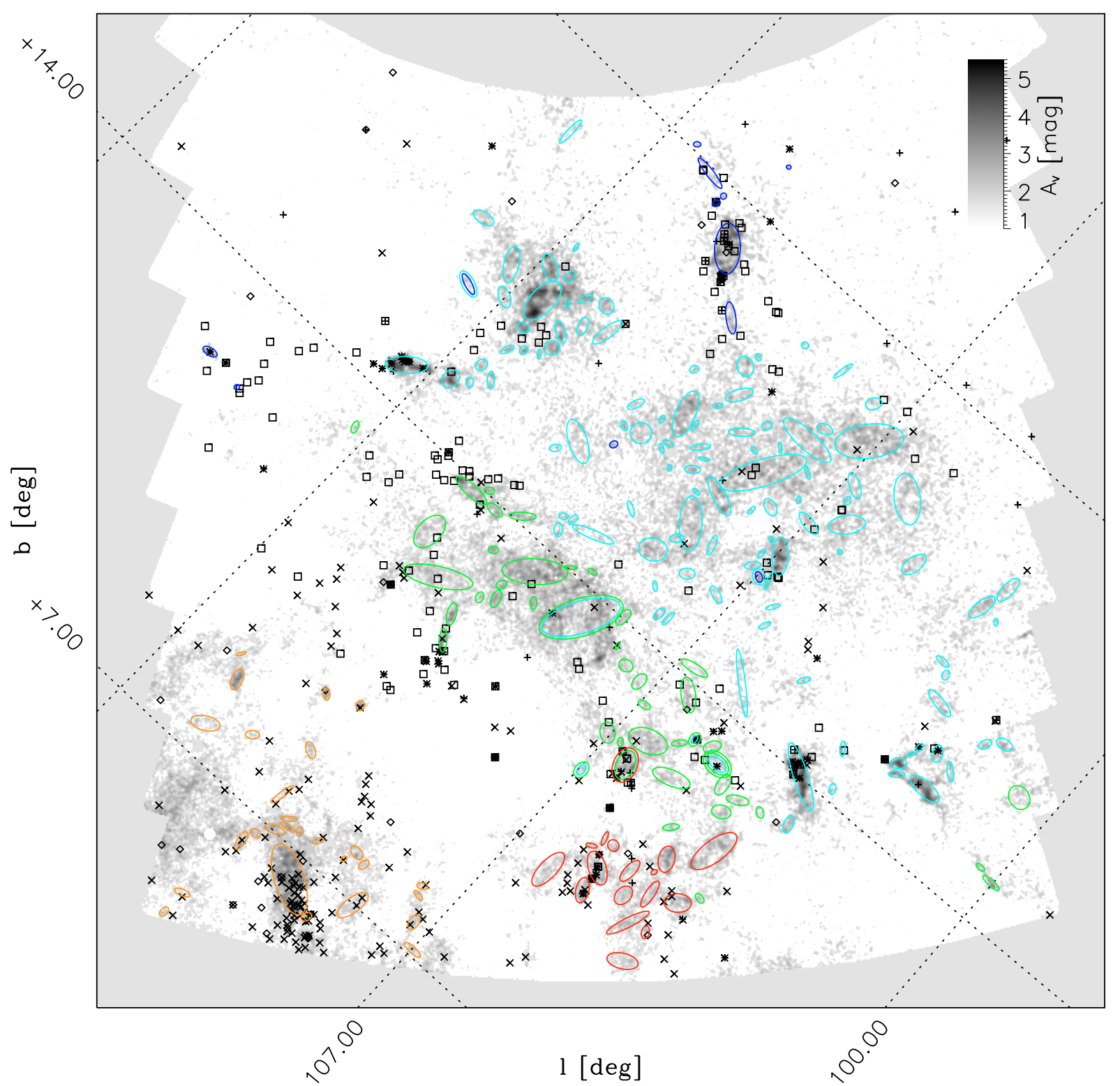

Fig. 1. Ellipses fitted to our clouds plotted on extinction maps. Colours denote distance zones: blue: $\approx 200 \mathrm{pc}$, cyan: $\approx 300 \mathrm{pc}$, green: $\approx 400 \mathrm{pc}$, orange: $\approx 650 \mathrm{pc}$, and red: $\approx 900 \mathrm{pc}$. YSO candidates from the 2MASS (diamonds), IPSC (crosses), IFSC (plus signs), and H $\alpha$ (squares) surveys by Kun (1998) are also plotted. Detailed data concerning the clouds are listed in Table A.1.

and coincidences in positions of different types of YSO candidates were taken into account.

\section{Results}

\subsection{Comparison of $A_{\mathrm{V}}^{\mathrm{USNO}}$ and $A_{\mathrm{V}}^{2 \mathrm{MASS}}$}

As was described in Sect. 2, extinction mapping based on optical data results in underestimated values for the most opaque peaks because of the low number density of stars at the limiting magnitude. According to Fig. $2, A_{\mathrm{V}}^{\mathrm{USNO}}$ clearly breaks down above $2.5 \mathrm{mag}$, while $A_{\mathrm{V}}^{2 \mathrm{MASS}}$ can reliably trace the extinction up to $\approx 8$ mag. This may cause significant differences, but only in the vicinity of opaque peaks, and this explains the difference in values $A_{\mathrm{V} \text {,peak }}^{\mathrm{USNO}}$ and $A_{\mathrm{V} \text {,peak }}^{2 \mathrm{MASS}}$ in Table 2 .

We checked the linear correlation between the values of $A_{\mathrm{V}}^{\mathrm{USNO}}$ and $A_{\mathrm{V}}^{2 \mathrm{MASS}}$ in the range from 0 to $2.5 \mathrm{mag}$, where both were found to be reliable. The correlation coefficient is 0.59 , and the slope of the fitted line is $\Delta A_{\mathrm{V}}^{\mathrm{USNO}} / \Delta A_{\mathrm{V}}^{2 \mathrm{MASS}}=0.95$. The scatter of the data points around the fitted line is 0.42 . The value of the slope may be affected by the combination of the following effects:

i) the uncertainties in statistical foreground correction at low extinction levels, coming from the fact that to distinguish foreground stars is not straightforward without knowing distance values, and

ii) the local changes in the actual value of the total-to-selective extiction ratio, which may depend on the extinction level, and affects the actual values of coefficients that describe the relationships between NIR colour excesses and the visual extinction.

The data sets used do not give sufficient information to calculate these effects separately, mainly because they remain under the error level. 


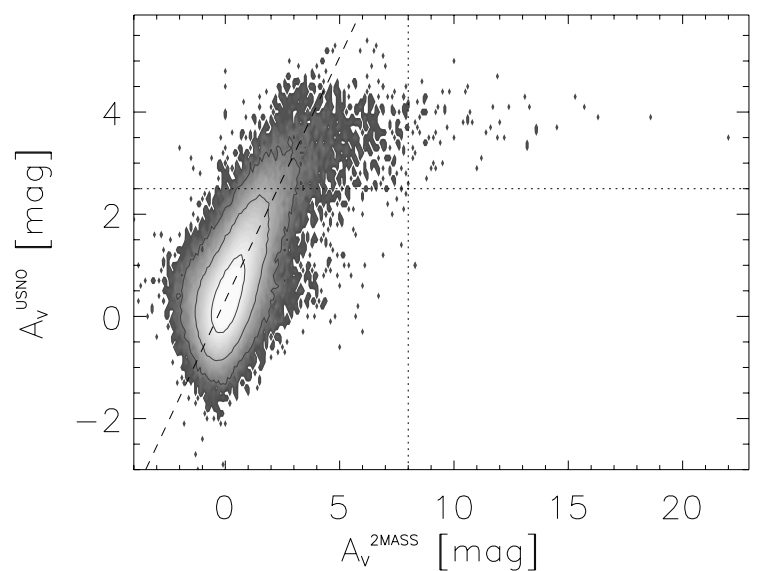

Fig. 2. Density plot of pixel values of $A_{\mathrm{V}}^{\mathrm{USNO}}$ and $A_{\mathrm{V}}^{2 \mathrm{MASS}}$ maps smoothed to the same resolution. Lighter grey tones imply a higher density of data points. Contours represent the data point densities $10^{3}, 10^{4}$, and $10^{5} \mathrm{mag}^{-2}$, respectively. The result of the linear fit to the values of the pixel pairs is also plotted (dashed line). The diagram shows good linear correlation in the extinction for the values $A_{\mathrm{V}}^{\mathrm{USNO}}<2.5 \mathrm{mag}$. The reliability limits of values are also plotted (dotted lines).

The definition of clouds and the description of their shapes is possible using either of the two maps, since the boundary levels are expected to be in the reliable range. Only a relatively small number of data points $(<1 \%)$ are situated above 2.5 mag and fail to follow the correlation. As is usually done, we used the $A_{\mathrm{V}}^{\mathrm{USNO}}$ map for this purpose.

\subsection{Global description and cloud complexes}

The distribution of extinction $A_{\mathrm{V}}^{\mathrm{USNO}}$ is shown in Fig. 3. The mean extinction in the field is $\frac{A_{\mathrm{V} \text {,field }}^{\mathrm{USNO}}}{2}=0.6 \mathrm{mag}$ with an $\mathrm{rms}$ fluctuation of $\sigma_{A}\left(s=3^{\prime}\right)=0.7 \mathrm{mag}$ (i.e. the standard deviation for the sample of regions of a typical scale, comparable to the resolution $s=3^{\prime}$ ). The highest extinction value of the whole region of $A_{\mathrm{V}, \text { max }}^{\mathrm{USNO}}=5.5 \mathrm{mag}$ was found in the dark cloud LDN 1241 (G113.4+17.1). The extinction distribution shows structures on a $5^{\circ}$ scale. The large-scale characteristics of $A_{\mathrm{V}}^{\mathrm{USNO}}$ were derived by calculating the mean extinction in elliptical moving windows with typical axis lengths of $5^{\circ}$. We generated a sample of mean extinction values in randomly placed ellipses. The mean value and the rms fluctuation of this sample were $\overline{A_{\mathrm{V} \text {,ell }}^{\mathrm{USNO}}}=0.6 \mathrm{mag}$ and $\sigma_{A}\left(s=5^{\circ}\right)=0.3 \mathrm{mag}$.

We identified four voids, as can be seen in Fig. 3, with an average diameter of $4.5^{\circ}$. These are regions where the mean extinction drops significantly, since its typical value is $\overline{A_{\mathrm{V}, \text { void }}^{\mathrm{USNO}}} \approx$ $0.3 \mathrm{mag}$.

Eight large cloud complexes (Fig. 3) were identified where $A_{\mathrm{V}}^{\mathrm{USNO}}$ was significantly higher than the average. The typical average extinction in the complexes is $\overline{A_{\mathrm{V}, \mathrm{cpl}}^{\mathrm{USNO}}} \approx 0.9 \mathrm{mag}$. Table 1 lists the basic parameters of these 8 cloud complexes, where the columns are: (1) name, (2)-(3) galactic coordinates of the centre position, (4) area in square degrees, (5) mean extinction of the complexes (the voids excluded) with rms fluctuation, (6) mean extinction in the complexes where $A_{\mathrm{V}}^{\mathrm{USNO}}>0.6 \mathrm{mag}$ with rms fluctuation, and (7) peak extinction $A_{\mathrm{V} \text {,peak }}^{2 \text { MASS }}$.

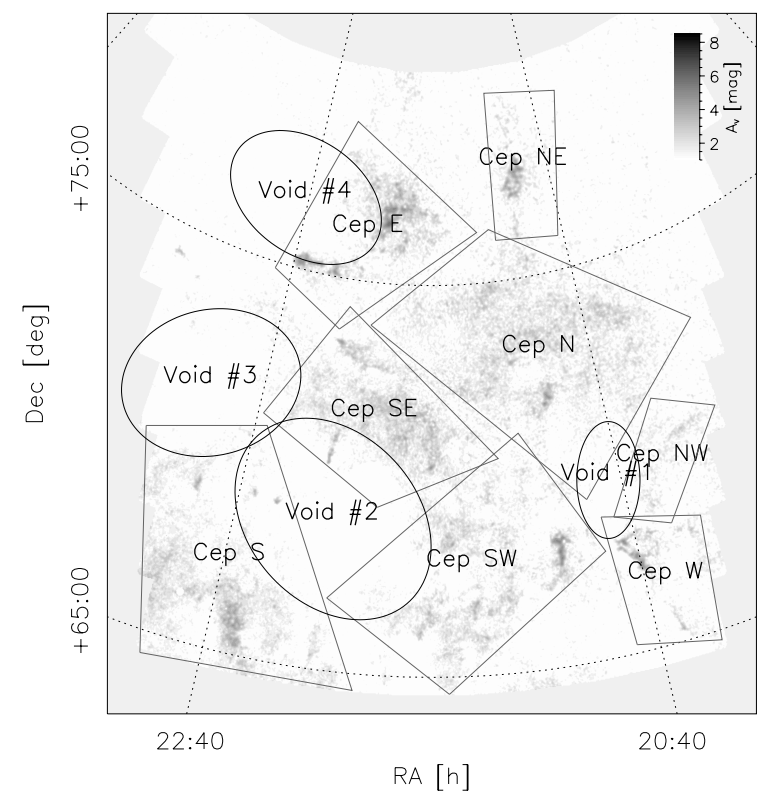

Fig. 3. Distribution of extinction $A_{\mathrm{V}}^{\mathrm{USNO}}$ in the Cepheus region. The scale of grey tones is shown on the bar (equatorial orientation, corners: $22^{\mathrm{h}} 58^{\mathrm{m}}, 62^{\circ} 44^{\prime} ; 20^{\mathrm{h}} 22^{\mathrm{m}}, 62^{\circ} 44^{\prime} ; 18^{\mathrm{h}} 29^{\mathrm{m}}, 78^{\circ} 4^{\prime} ;$ and $\left.0^{\mathrm{h}} 51^{\mathrm{m}}, 78^{\circ} 4^{\prime}\right)$. The boundaries of complexes (polygons) and voids (ellipses) are overlaid. The parameters of these structures are listed in Tables 1, 5, and 6.

Table 1. Basic parameters of the 8 cloud complexes.

\begin{tabular}{|c|c|c|c|c|c|c|}
\hline $\begin{array}{l}\text { Cep } \\
\text { id. } \\
\text { (1) }\end{array}$ & (2) & (3) & $\begin{array}{r}S \\
{\left[\square^{\circ}\right]} \\
(4)\end{array}$ & $\begin{array}{r}\overline{A_{\mathrm{V}}^{\mathrm{USNO}}} \\
{[\mathrm{mag}]} \\
(5)\end{array}$ & $\begin{array}{r}A_{\mathrm{V}, 0.6}^{\mathrm{USNO}} \\
{[\mathrm{mag}]} \\
(6)\end{array}$ & $\begin{array}{r}A_{\mathrm{V}, \mathrm{peak}}^{2 \mathrm{MASS}} \\
{[\mathrm{mag}]} \\
(7)\end{array}$ \\
\hline $\mathrm{N}$ & $107^{\circ} .9$ & 17.4 & 31 & $1.1 \pm 0.6$ & $1.3 \pm 0.5$ & 5.7 \\
\hline NE & $111^{\circ} .7$ & 20.6 & 7 & $0.8 \pm 0.8$ & $1.3 \pm 0.7$ & 15.7 \\
\hline E & $113^{\circ} .7$ & 16.4 & 15 & $1.1 \pm 0.9$ & $1.4 \pm 0.8$ & 16.4 \\
\hline SE & $110^{\circ} .2$ & 13.1 & 16 & $1.2 \pm 0.7$ & $1.4 \pm 0.6$ & 10.0 \\
\hline S & $110^{\circ} 6$ & 7.7 & 28 & $0.9 \pm 0.7$ & $1.2 \pm 0.6$ & 11.7 \\
\hline SW & $105^{\circ} .5$ & $12^{\circ} .1$ & 25 & $0.9 \pm 0.7$ & $1.2 \pm 0.5$ & 22.0 \\
\hline $\mathrm{W}$ & $101^{\circ} .2$ & $15: 4$ & 8 & $0.7 \pm 0.7$ & $1.2 \pm 0.6$ & 10.0 \\
\hline NW & $103^{\circ} 3$ & 17.6 & 5 & $0.8 \pm 0.5$ & $1.1 \pm 0.4$ & 3.3 \\
\hline
\end{tabular}

\subsection{Dark clouds}

Census and distribution: As many as 208 dark clouds were localised, out of which 86 clouds have no previously catalogued associated cloud. The defined clouds cover $6.2 \%$ of the mapped area. Data of the whole cloud sample, along with the previously catalogued associated clouds, are listed in Tables A.1 and A.2, respectively. The average extinction in the clouds is $\overline{A_{\mathrm{V}, \text { cld }}^{\mathrm{USNO}}}=2.1 \mathrm{mag}$. The histogram of the peak extinction values is shown in Fig. 4. The peak extinction is over 4 mag in 11 clouds $(\approx 5 \%$ of all, see Table 2 ).

Morphology: Histograms of the morphological parameters are shown in Fig. 5. The distributions of some parameters were fitted with an $f(x)$ lognormal function:

$f(x)=\frac{\mathrm{e}^{-\frac{\left(\ln \frac{x-\theta}{m}\right)^{2}}{2 \sigma^{2}}}}{\sqrt{2 \pi} \sigma(x-\theta)}$

where $x=a, b / a, \mathrm{PA}$, irr. The distributions of $A_{\mathrm{V}}^{\mathrm{USNO}}$ (Fig. 4) and $a$ are cut off at their lower ends because of the completeness 
Table 2. ${ }^{1}$ Data of clouds with $A_{\mathrm{V}}^{\mathrm{USNO}}>4$ mag. The columns are: (1) cloud name, $(2,3)$ centre position in galactic frame, $(4,5)$ peak extinction derived from optical (USNO) and NIR (2MASS) data, $(6,7)$ apparent and linear size of major axis in arcmin and pc, respectively, (8) axis ratio, (9) position angle ccw from the direction of the northern galactic pole, (10) irregularity, (11) morphological class (see Sect.3.4), (12) distance, (13) cloud mass (see Sect. 3.5), (14) associated objects of the LDN and the Nagoya ${ }^{13}$ CO cloud catalogues, and (15) number of YSO candidates associated with the clouds. More detailed data of the whole cloud sample are presented in Table A.1.

\begin{tabular}{|c|c|c|c|c|c|c|c|c|c|c|c|c|c|}
\hline Name & $\begin{array}{r}l \\
{\left[{ }^{\circ}\right]}\end{array}$ & $\begin{array}{r}b \\
{\left[{ }^{\circ}\right]}\end{array}$ & $\begin{array}{l}A_{\mathrm{V}, \text { peak }}^{\mathrm{USNO}} \\
{[\mathrm{m}}\end{array}$ & $\begin{array}{l}4_{\mathrm{V}, \text { peak }}^{2 \text { MASS }} \\
\text { g] }\end{array}$ & $\begin{array}{r}a \\
{[']}\end{array}$ & $\begin{array}{r}a \\
{[\mathrm{pc}]}\end{array}$ & $b / a$ & $\begin{array}{l}\mathrm{PA} \\
\left.{ }^{\circ}\right]\end{array}$ & irr $c l$ & $\begin{array}{l}{ }^{2} d \\
{[\mathrm{pc}]}\end{array}$ & $\begin{array}{r}M \\
{\left[M_{\odot}\right]}\end{array}$ & ${ }^{3}$ Associated clouds & $N$ \\
\hline (1) & (2) & (3) & (4) & (5) & (6) & (7) & (8) & (9) & (10) (11) & (12) & (13) & (14) & (15) \\
\hline $\mathrm{G} 102.0+15.3$ & 102.06 & 15.30 & 4.6 & 8.8 & 40.7 & $\overline{5.3}$ & $\overline{0.4}$ & 90 & 0.31 & ${ }^{c} 325$ & 218 & LDN 1147,1148 & 1 \\
\hline G102.7+15.3 & $102^{\circ} .73$ & 15.34 & 4.6 & 10.0 & $17: .5$ & 2.3 & 0.7 & 130 & $0.32 h t$ & ${ }^{c} 325$ & 71 & LDN 1155, CO 8 & 0 \\
\hline G103.9+14.0 & 103.90 & 14.01 & 4.7 & 22.0 & $77: 5$ & 10.1 & 0.2 & 60 & $0.42 h t$ & ${ }^{c} 288$ & 321 & LDN 1172,1174, CO 14 & 7 \\
\hline G106.9+16.7 & 106.93 & 16.72 & 4.2 & 5.1 & $42 ! 2$ & 3.7 & 0.4 & 40 & 0.14 & ${ }^{a} 300$ & 181 & & 0 \\
\hline G108.8+13.5 & $108^{\circ} 86$ & 13.51 & 4.3 & 7.7 & $87: 7$ & 7.7 & 0.4 & 150 & 1.37 & ${ }^{a} 300,{ }^{b} 400$ & 690 & $\mathrm{CO} 43$ & 2 \\
\hline G109.4+06.5 & 109.45 & 6.56 & 4.2 & 11.7 & 88.8 & 15.3 & 0.4 & 60 & 0.20 & ${ }^{b} 650$ & 3165 & LDN 1213,1214, CO 48 & 21 \\
\hline G111.6+20.1 & 111.64 & $20^{\circ} 14$ & 4.2 & 15.7 & $55^{\prime} .9$ & 3.3 & 0.5 & 40 & 0.23 & ${ }^{a} 200$ & 182 & LDN 1228, CO 66 & 10 \\
\hline G111.9+13.8 & 111.94 & 13.84 & 4.1 & 10.0 & 43.0 & 5.0 & 0.3 & 90 & 0.49 & ${ }^{b} 400$ & 223 & CO 69 & 0 \\
\hline G113.4+17.1 & $113: 46$ & 17.19 & 5.5 & 10.1 & $52 ! 8$ & 4.6 & 0.5 & 0 & 0.40 & ${ }^{a} 300$ & 437 & $\mathrm{CO} 75$ & 0 \\
\hline G113.6+15.0 & $113^{\circ} 66$ & $15^{\circ} .04$ & 4.4 & 12.2 & $21: 8$ & 1.9 & 0.8 & 0 & 0.25 & ${ }^{a} 300$ & 100 & LDN 1247 & 1 \\
\hline G114.4+14.6 & $114^{\circ} 42$ & 14.67 & 4.5 & 16.4 & $46^{\prime} 0$ & 4.0 & 0.4 & 130 & $0.25 h t$ & ${ }^{a} 300$ & 246 & LDN 1251, CO 79 & 9 \\
\hline
\end{tabular}

${ }^{1}$ A more complete list of cloud data with associated clouds, according to a number of catalogues, is presented in Table A.2.

${ }^{2}$ Distance references: ${ }^{a}$ Kun (1998), ${ }^{b}$ Racine (1968), and ${ }^{c}$ Straižys et al. (1992).

${ }^{3}$ SIMBAD identifier of clouds referred to as $\mathrm{CO} n$ is [YDM] CO $n$.
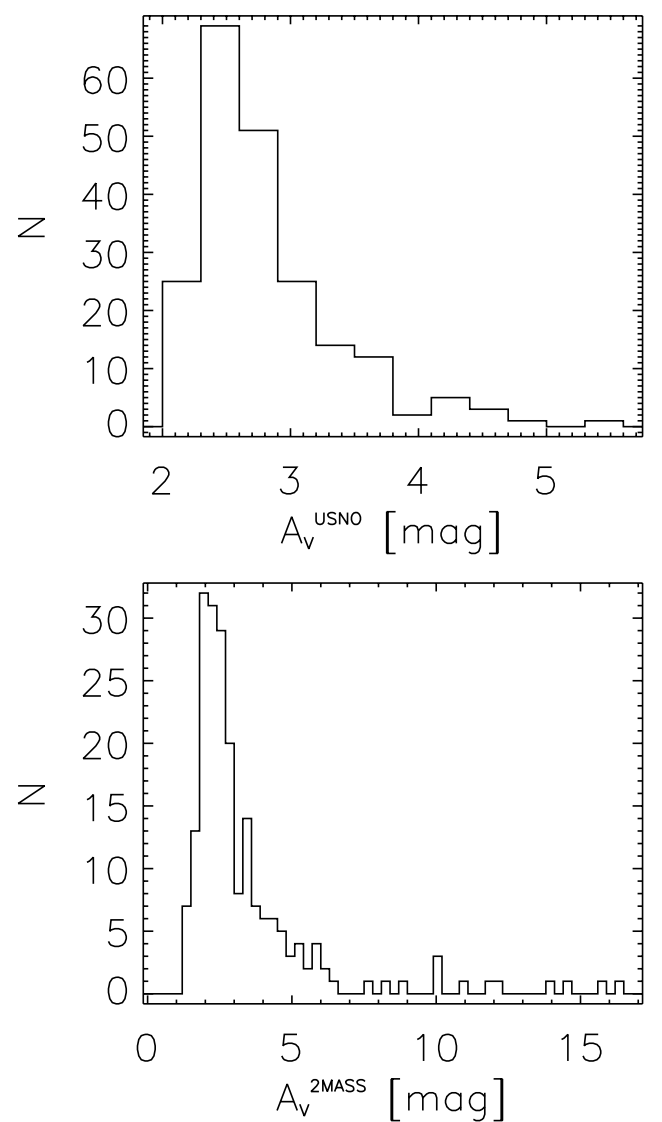

Fig. 4. Histograms of peak extinctions $A_{\mathrm{V}}^{\mathrm{USNO}}$ and $A_{\mathrm{V}}^{2 \mathrm{MASS}}$ in the 208 clouds.

limit of the sample. The shapes of clouds in different complexes do not differ significantly. We note, in particular, that the values of $a$ and $b / a$ parameters do not vary significantly from one complex to another.
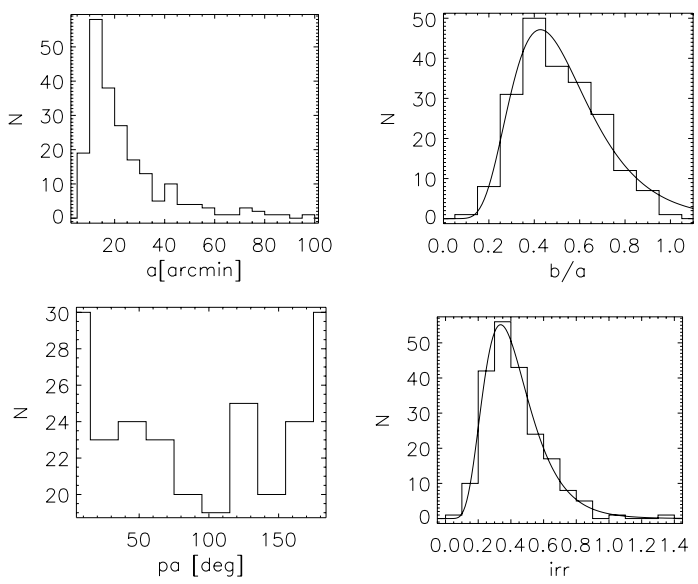

Fig. 5. Histograms of morphological parameters: major axis length $a$, axis ratio $b / a$, position angle PA and irregularity irr. Overplotted solid curves indicate the fitted lognormal distributions. The parameters of the lognormals are seen in Table 3.

\subsection{Morphological classification}

As was described above, the defined parameters may help us find morphological features in the clouds of our sample. Based on a visual inspection of the sample of clouds, we selected 12 globular clouds and another 12 head-tail-shaped clouds. We examined the morphological parameters of the selected clouds, searching for some quantitative difference between the two morphological groups. Though visually recognized globular and head-tail clouds have different mean values of the morphological parameters, due to the scatter of the values, this difference is not certain enough to be the basis of a quantitative classification scheme. Therefore, the subsamples of clouds with globular and headtail morphology were defined only through visual inspection. Examples of such clouds are shown in Fig. 6.

The first example, G116.3+12.2, is one of the most clearly separated globular clouds. It is situated apart from groups of clouds, outside the complexes, in a low extinction region. The cloud has a moderate peak extinction $\left(A_{\mathrm{V}, \text { peak }}^{2 \mathrm{MASS}}=5.3 \mathrm{mag}\right)$ 
Table 3. Parameters of lognormal fits.

\begin{tabular}{lcccc}
\hline \hline & $m$ & $\theta$ & $\sigma$ & $P\left(\chi^{2}\right)$ \\
\hline$b / a$ & 0.51 & -0.02 & 0.38 & 0.819 \\
$i r r$ & 0.43 & -0.03 & 0.38 & 0.996 \\
\hline
\end{tabular}
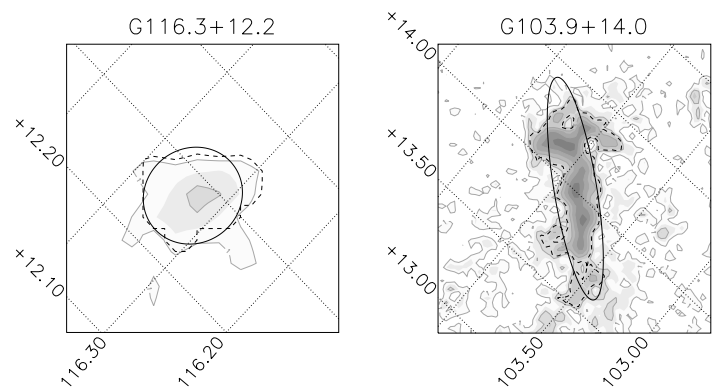

Fig. 6. Example clouds with globular and head-tail features. $A_{\mathrm{V}}^{\mathrm{USNO}}$ contours are drawn from $1 \mathrm{mag}$ to $5 \mathrm{mag}$ by $1 \mathrm{mag}$. Dashed curves indicate the cloud boundaries. Ellipses fitted to the clouds are also plotted.

Table 4. Cloud detectability limit of size and mass in the different distance layers.

\begin{tabular}{lrrrrrr}
\hline \hline Distance [pc] & 200 & 300 & 400 & 450 & 650 & 900 \\
\hline$a_{\min }[\mathrm{pc}]$ & 0.17 & 0.26 & 0.35 & 0.39 & 0.57 & 0.79 \\
$M_{\min }\left[M_{\odot}\right]$ & 0.6 & 1.3 & 2.3 & 2.8 & 5.9 & 11.4 \\
\hline
\end{tabular}

and a quite regular, round shape. We note that, due to its position, it is associated with the HII region LBN 116.20+12.34. The second example, G103.9+14.0, is situated at the edge of complex Cep W and is apparently connected to the other parts of the complex with a moderate extinction region, although separated from other neighboring complexes. The cloud itself has a high peak extinction $\left(A_{\mathrm{V}, \text { peak }}^{2 \mathrm{MASS}}=22.0 \mathrm{mag}\right)$ and a head-tail shape. The object includes the dark clouds LDN 1172 and LDN 1174, and it is associated with CO 14 (Yonekura et al. 1997).

We note that 5 of our globular and 4 of our head-tail clouds are newly discovered.

\subsection{Mass determination}

The total mass integrated over the whole region is $M_{\mathrm{tot}}=$ $2.1 \times 10^{5} M_{\odot}$, assuming a mean distance of $400 \mathrm{pc}$. The cloud detectability limits (i.e. a possible cloud with a peak at $2 \sigma$ signal level, and an angular size of $3^{\prime}$, which is the mean resolution at this signal level) of size and mass in the different layers were determined and listed in Table 4. The upper limit for total mass in undetectable clouds is $M_{\mathrm{u}}=2.9 \times 10^{4} M_{\odot}$, assuming a mean distance of $400 \mathrm{pc}$. We determined the mass of each individual cloud. The total mass in clouds is $M_{\mathrm{t}, \mathrm{cl}}=3.1 \times 10^{4} M_{\odot}$. As much as $21 \%$ of this total mass is comprised in the 11 most opaque clouds with peak $A_{\mathrm{V}}^{\mathrm{USNO}}$ extinction higher than 4 mag. The total mass of individual complexes, calculated using the typical distances of the contained clouds, and the ratio of the mass contained in clouds are listed in Table 5. The total mass in globular and head-tail clouds are 286 and $2515 M_{\odot}$, respectively. Compared to the ${ }^{13} \mathrm{CO}$ cloud groups presented by Yonekura et al. (1997), our complexes have somewhat higher masses, although the boundaries of the two arrangements do not exactly coincide.
Table 5. Derived parameters of complexes. The columns are: (1) name, (2) mean of cloud peak $A_{\mathrm{V}}^{2 \mathrm{MASS}}$ extinctions in the complex [mag], (3) average of cloud mean $A_{\mathrm{V}}^{\mathrm{USNO}}$ extinctions in the complex [mag], (4) total mass estimated from extinction $\left[M_{\odot}\right],(5)$ ratio of cloud mass in the complex to its total mass [\%], (6) star-forming efficiency [\%], and (7) mean temperature of total area inside the complexes $[\mathrm{K}]$.

\begin{tabular}{|c|c|c|c|c|c|c|}
\hline $\begin{array}{l}\text { Cep } \\
\text { id. } \\
\text { (1) }\end{array}$ & $\begin{array}{r}\overline{A_{\mathrm{V}, \text { peak }}^{2 \text { MASS }}} \\
{[\mathrm{mag}]} \\
(2)\end{array}$ & $\begin{array}{r}A_{\mathrm{V}, \text { mean }}^{\mathrm{USNO}} \\
{[\mathrm{mag}]} \\
(3)\end{array}$ & $\begin{array}{r}M^{1} \\
{\left[M_{\odot}\right]} \\
(4)\end{array}$ & $\begin{array}{r}\eta_{c f} \\
{[\%]} \\
(5)\end{array}$ & $\begin{array}{r}\eta_{s f} \\
{[\%]} \\
(6)\end{array}$ & $\begin{array}{r}\overline{T_{\mathrm{DC}}^{c p l}} \\
{[\mathrm{~K}]} \\
(7)\end{array}$ \\
\hline $\mathrm{N}$ & $2.7(0.9)$ & $2.0(0.4)$ & 14698 & 26 & 0.06 & $14.7(1.1)$ \\
\hline $\mathrm{NE}$ & $5.7(5.1)$ & $2.5(0.8)$ & 1016 & 24 & 2.50 & $14.6(1.1)$ \\
\hline E & $4.5(3.5)$ & $2.6(0.8)$ & 5753 & 29 & 0.30 & $14.2(1.0)$ \\
\hline SE & $3.2(2.0)$ & $2.3(0.5)$ & 12531 & 19 & 0.06 & $15.6(1.0)$ \\
\hline $\mathrm{S}$ & $2.6(2.2)$ & $2.3(0.7)$ & 50592 & 12 & 0.26 & $18.2(2.0)$ \\
\hline SW & $3.5(3.3)$ & $2.0(0.6)$ & 44695 & 30 & 0.09 & $17.0(1.8)$ \\
\hline W & $5.0(2.7)$ & $2.3(0.8)$ & 5909 & 21 & 0.12 & $14.1(0.6)$ \\
\hline NW & $2.5(0.7)$ & $1.7(0.3)$ & 1713 & 14 & 0.00 & $14.5(0.8)$ \\
\hline
\end{tabular}

1 Distances used for individual complexes: NE: $200 \mathrm{pc} ; \mathrm{NW}, \mathrm{N}$, E: 300 pc; SE: 400 pc; W: $450 \mathrm{pc}$; and S, SW: 650 pc.

\subsection{FIR properties}

Global characteristics: The FIR properties were examined using the methods proposed by Tóth et al. (2000). The slope $I(100) / I(170)=0.56$ was defined with a bisector linear fit in the $I(100)$ versus $I(170)$ scatterplot, as shown in Fig. 7. The $T_{\mathrm{DC}}^{\text {avg }}=16.5 \mathrm{~K}$ average dust colour temperature is defined using a modified Planck function with the $\beta=2$ emissivity law. Subregions with average FIR colours were defined by the relation $0.56 \times I(170)-11.19<I(100)<0.56 \times I(170)-21.19$. We assume that any significant deviation from the rms fit is due to a different (either higher or lower) colour temperature. Subregions with average, higher, and lower colour temperatures occupy $51 \%, 46 \%$, and $3 \%$, respectively, of the total area covered by ISO data (Fig. 7).

The subregions with average colour temperatures outline the large cloud complexes. The inter-complex areas, including the 4 voids (low extinction regions), are warmer, as can be seen in Fig. 7. Additionally, a few small warm spots appear inside the cloud complexes. The cold regions are confined to several small spots within the cloud complexes. The dependence of the average dust colour temperature on the galactic latitude was also investigated. The region was divided into $\Delta b=3^{\circ}$ wide stripes, and the average colour temperature was derived for each stripe from the slope of the linear fits to the $I(100)$ and $I(170)$ data points. The variation of the average colour temperature $T_{\mathrm{DC}}^{b}$ with the galactic latitude $b$ is plotted in Fig. 8, clearly demonstrating the linear correlation over the interval $3^{\circ}<b<18^{\circ}$. The FIR colour temperature appears to decrease with increasing galactic latitude for points both inside and outside the clouds, in the range of $3^{\circ}<b<18^{\circ}$ as:

$$
T_{\mathrm{DC}}^{b}=19.8[\mathrm{~K}]-0.30[\mathrm{~K} / \mathrm{deg}] \times b .
$$

Large structures: We investigated the cold, average, and warm components for each of the eight large cloud complexes, as well as for the four voids. The complexes and their derived parameters are listed in Table 5. The 4 voids are listed in Table 6. The average colour of the cloud complexes depends mainly on their galactic latitude. The variability of colour temperature within a single complex is largest in complex Cep $\mathrm{S}$ and smallest in Cep W. 

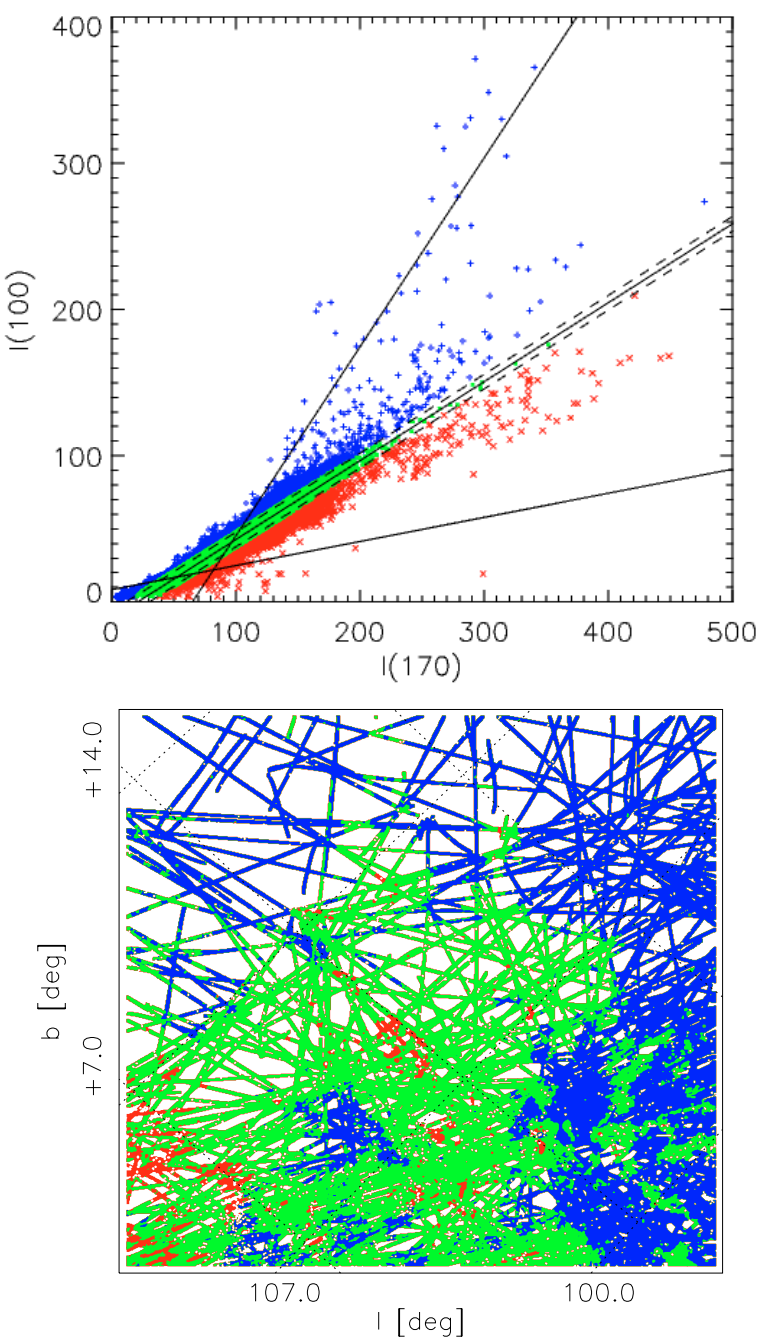

Fig. 7. Top: plot of $I(100)$ versus $I(170)$ with the fitted (solid) line representing the mean temperature of the region $\left(T_{\mathrm{DC}}^{\text {avg }}=16.5 \mathrm{~K}\right)$. Dashed lines define the range within $\pm 5 \mathrm{MJy} / \mathrm{sr}$ in $I(100)$ from the fit, and the data points in the range are denoted with green symbols. Data above and below this range represent somewhat higher (blue symbols) and lower (red symbols) temperatures, with fitted values of $25.1 \mathrm{~K}$ and $13.3 \mathrm{~K}$, respectively. Bottom: a map of the region was also drawn using the same colour codes, i.e. subregions having average temperature were filled with green, while higher and lower temperature subregions were filled with blue and red colours, respectively. However, the field was not completely covered by the ISO $170 \mu \mathrm{m}$ serendipity survey. Positions that were not observed remain white on this map since the temperature could not be calculated for these areas.

We have also checked the $I(12) / I(100)$ (see Fig. 9) and $I(12) / I(170)$ colours. The $12 \mu \mathrm{m}$ surface brightness $I(12)$ correlates well with $I(100)$ and $I(170)$; however, the slopes of the scatterplots depend on the galactic latitude. The northern and eastern walls of void \#2 clearly show a relative excess of $I(12)$.

Dark clouds: The $I(100)$ versus $I(170)$ scatterplot of all nonstellar positions inside dark clouds in Fig. 10 indicates an average colour temperature of $T_{\mathrm{DC}}^{\mathrm{cla}} \approx 16.5 \mathrm{~K}$. The fit to the points of regions warmer than the average gives $T_{\mathrm{DC}}^{\mathrm{clw}} \approx 25.1 \mathrm{~K}$. These appear to be the positions at the luminous parts of the headtail clouds. The fit to the colder regime gives $T_{\mathrm{DC}}^{\mathrm{clc}} \approx 13.3 \mathrm{~K}$. Positions with the highest FIR brightness and average colour temperature are mainly located in unclassified clouds. Globular

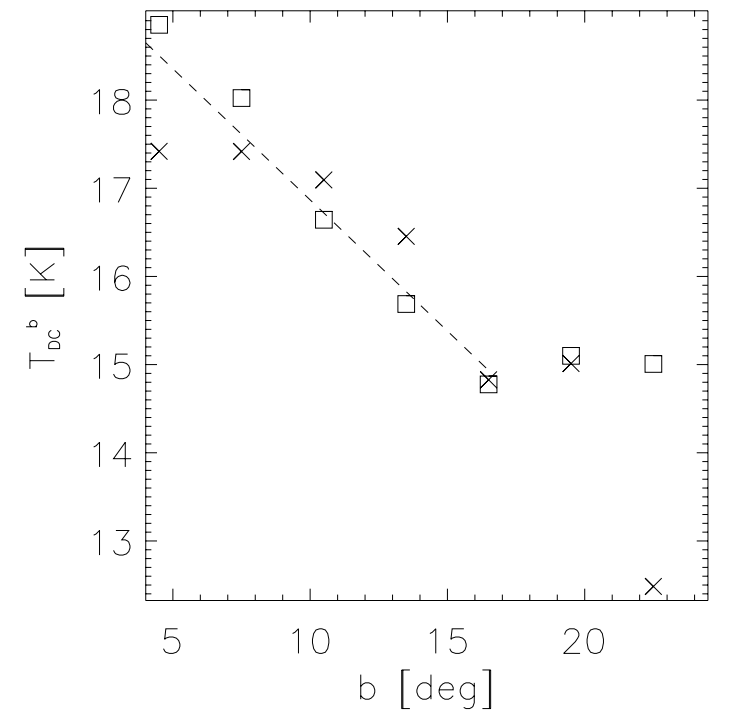

Fig. 8. Mean colour temperature versus galactic latitude ranges from $\left[3^{\circ}, 6^{\circ}\right]$ to $\left[21^{\circ}, 24^{\circ}\right]$. Squares denote data outside the clouds, and crosses denote data inside the clouds. The dashed line $\left(T_{\mathrm{DC}}^{\mathrm{b}}=19.8[\mathrm{~K}]-\right.$ $0.30[\mathrm{~K} / \mathrm{deg}] \cdot b$ ) was fitted to the data points below $b=18^{\circ}$.

Table 6. Parameters of the voids in the region. The columns are: (1) number, $(2,3)$ galactic coordinates, $(4,5)$ semimajor and semiminor axis [deg], (6) position angle ccw from the direction of the northern galactic pole, (7) $\overline{A_{\mathrm{V}, \text { void }}^{\mathrm{USNO}}}$ average extinction [mag], (8) $\overline{A_{\mathrm{V},(3 / 4)}^{\mathrm{USNO}}}$ average extinction calculated for the inner part of the void, using the defined ellipses with reduced axes by a factor of $3 / 4$, and (9) average FIR colour temperature.

\begin{tabular}{rrrrrrrrr}
\hline \hline $\begin{array}{r}\text { void } \\
\text { no. }\end{array}$ & $l$ & $b$ & $r_{\text {maj }}$ & $r_{\text {min }}$ & PA & $\begin{array}{r}\overline{A_{\mathrm{V}, \text { void }}^{\mathrm{USN}}} \\
{[\mathrm{mag}]}\end{array}$ & $\begin{array}{r}\overline{A_{\mathrm{V}, \text { in }}^{\mathrm{USN}}} \\
{[\mathrm{mag}]}\end{array}$ & $\begin{array}{r}\overline{\mathrm{DC}, \text { in }} \\
{[\mathrm{K}]}\end{array}$ \\
$(1)$ & $(2)$ & $(3)$ & $(4)$ & $(5)$ & $(6)$ & $(7)$ & $(8)$ & $(9)$ \\
\hline$\# 1$ & 104.1 & 16.2 & 1.50 & 0.88 & $43^{\circ}$ & $0.4 \pm 0.4$ & $0.3 \pm 0.4$ & 15.4 \\
$\# 2$ & 109.2 & 10.4 & 3.00 & 2.25 & $-83^{\circ}$ & $0.3 \pm 0.5$ & $0.1 \pm 0.5$ & 16.6 \\
$\# 3$ & $114^{\circ} 1$ & 10.6 & 10.25 & 0.94 & $-37^{\circ}$ & $0.2 \pm 0.4$ & $0.3 \pm 0.4$ & 15.2 \\
$\# 4$ & $115^{\circ} 6$ & 15.8 & 2.25 & 1.50 & $-73^{\circ}$ & $0.3 \pm 0.6$ & $0.2 \pm 0.5$ & 14.0 \\
\hline
\end{tabular}

clouds are situated mostly in the faint part of the plot. The region $I(100)>150 \mathrm{MJy} / \mathrm{sr}$ is mainly occupied by head-tail class clouds. The colour temperature, however, varies considerably inside clouds, as well. To give an example, the FIR properties of G103.9+14.0 are shown in Fig. 11. We note that all positions of G103.9+14.0, faint in FIR, appear to be cold. The head part with several YSOs around ammonia cores of L1174 (Benson \& Myers 1989), shows a relatively high temperature, while the tail part, with one YSO associated with the ammonia core L1172A (Benson \& Myers 1989), shows a low FIR colour temperature.

We determined the minimum temperatures $T_{\mathrm{DC}}^{\mathrm{min}}$ of all clouds for which $170 \mu \mathrm{m}$ ISOSS data were available. The values range between $13 \mathrm{~K}$ and $18 \mathrm{~K}$. The minimum temperatures of clouds decrease with both increasing opticaland NIR-based extinction. $T_{\mathrm{DC}}^{\mathrm{min}}$ shows a clear linear decrease with galactic latitude (see Fig. 12), according to the formula $T_{\mathrm{DC}}^{\min }(b)=16.63[\mathrm{~K}]-0.09[\mathrm{~K} / \mathrm{deg}] \cdot b$. The correlation coefficient is -0.69 . We also checked the correlation between the latitude and the optical extinction. In this case, the correlation coefficient was only -0.03 , i.e. there is practically no correlation. A corrected temperature has been defined by removing the dependency on galactic latitude, as: $\Delta T_{\mathrm{DC}}^{\min }=T_{\mathrm{DC}}^{\min }-T_{\mathrm{DC}}^{\mathrm{min}}(b)$. 


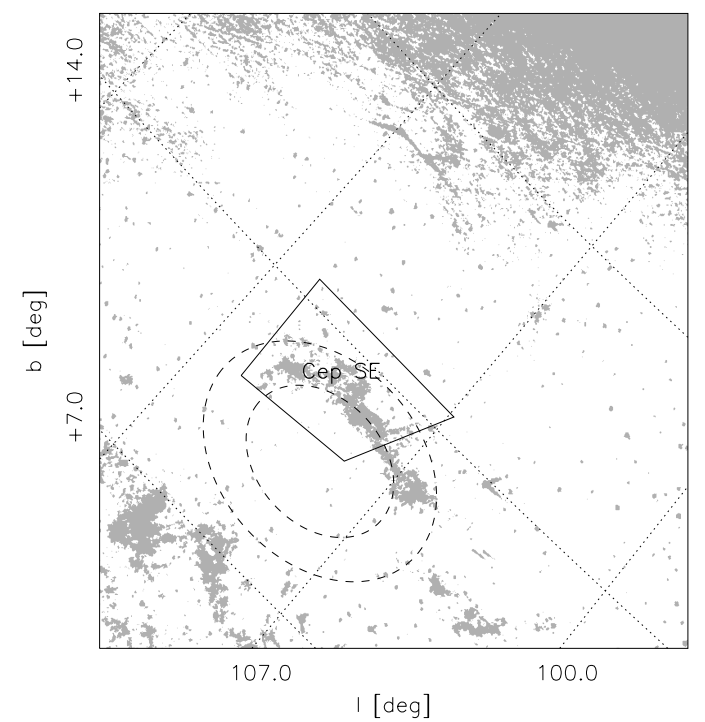

Fig. 9. Two-level map to visualise regions with $12 \mu \mathrm{m}$ excess from $I(12)$ and $I(100)$ data above and below $I(12)=5.27 \times 10^{-1}+3.27 \times 10^{-2} I(100)$. Cep SE (polygon) and GIRL G109+11 (dashed double ellipse) are also plotted.

We plotted $\Delta T_{\mathrm{DC}}^{\mathrm{min}}$ versus $A_{\mathrm{V}}^{\mathrm{USNO}}$ (Fig. 12). This figure shows that $\Delta T_{\mathrm{DC}}^{\mathrm{min}}$ also decreases with increasing optical extinction. A relation between the $\Delta T_{\mathrm{DC}}^{\mathrm{min}}$ and the masses of the clouds $M$ is shown in the right panel of Fig. 12. At low mass values, most of the clouds have relatively high $\left(\overline{\Delta T_{\mathrm{DC}}^{\min }}>0\right)$, while at higher mass values clouds mostly have relatively low minimum temperature $\left(\overline{\Delta T_{\mathrm{DC}}^{\min }}<0\right)$. The transition seems to be between 50 and $100 M_{\odot}$.

FIR colour indices of the dark clouds: We also derived background-corrected FIR colour indices $\Delta I(100) / \Delta I(60)$ and $\Delta I(170) / \Delta I(100)$ (see Fig. 13). Background values of surface brightness were defined as the lowest emission at the given FIR band in the environment of the cloud within a radius of 1.2 times the square root of the cloud area. Background subtraction was carried out at each wavelength separately. For globular clouds, $\Delta I(100) / \Delta I(60)$ mostly remains below 10 , although it can exceed 20 for head-tail clouds. Apparently, the background-corrected FIR colours of the head-tail clouds vary much more than those of the globular clouds.

\section{Discussion}

\subsection{Morphology}

In former cloud catalogues (Barnard 1927; Lynds 1962), the clouds were defined visually and morphological characteristics were ignored. As was mentioned in Sect. 2, the use of a constant contour level to define clouds would strongly bias the sample of clouds. Choosing the boundary level at $A_{\mathrm{V}}^{\mathrm{USNO}}=2.0 \mathrm{mag}$ corresponding to $2.5 \sigma$ with respect to the mean error in the field, the morphological parameters for most of the clouds that were visually defined as head-tail would not change significantly. In a few cases, however, the structure of a large cloud complex containing several density peaks would remain unresolved, while many of the isolated globules with peak extinction significantly above the noise level would contain only a few positions above the boundary level. Accordingly, their morphological examination would not be reasonable. Notable changes in the shape due
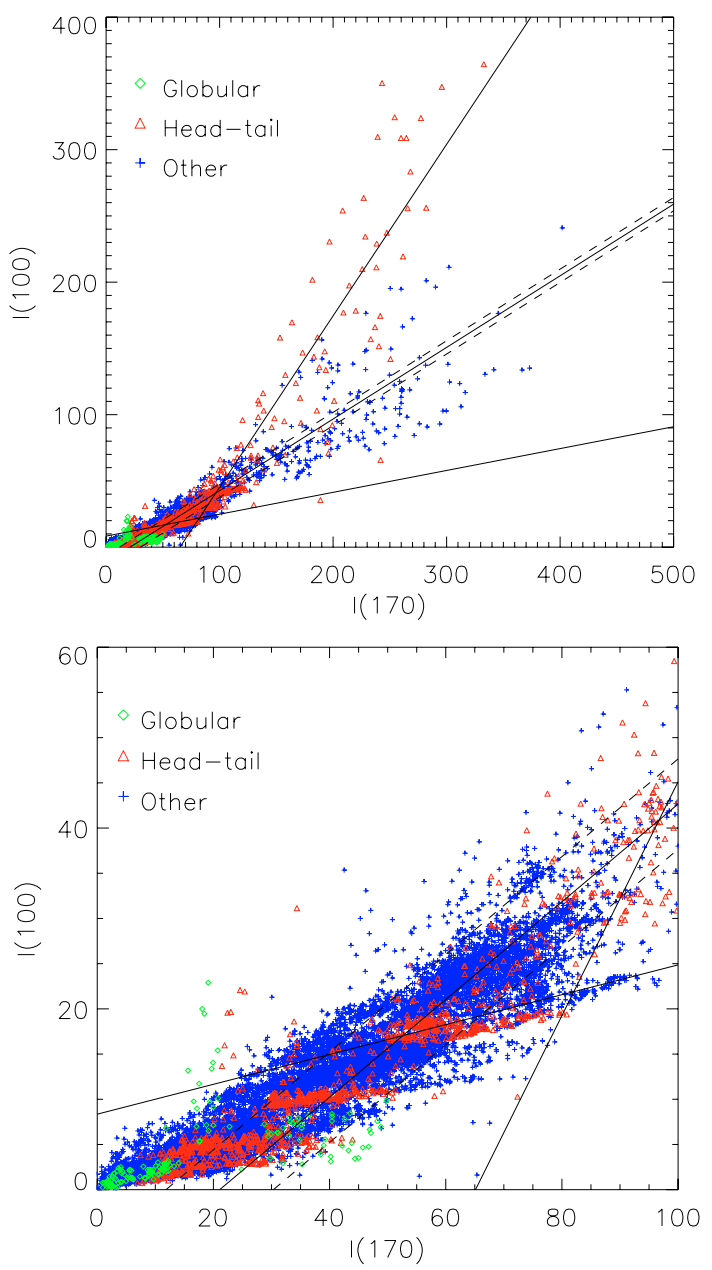

Fig. 10. Scatterplot of $I(100)$ versus $I(170)$ for all positions inside the dark clouds can be seen in the top panel. The same plot zoomed into the $I(170)$ range $[0 \mathrm{MJy} / \mathrm{sr}, 100 \mathrm{MJy} / \mathrm{sr}]$ is in the bottom panel. Linear fits are overlaid: data of the whole region (solid line), indicating $T_{\mathrm{DC}}^{\mathrm{avg}}=$ $16.5 \mathrm{~K}$, and a $\Delta I(100)= \pm 5 \mathrm{MJy} / \mathrm{sr}$ wide range (dashed line). Further solid lines were fitted to the colder (flat one) and warmer (steep one) branches representing $T_{\mathrm{DC}}^{\text {cold }}=13.3 \mathrm{~K}$ and $T_{\mathrm{DC}}^{\text {warm }}=25.1 \mathrm{~K}$ respectively. Colours and symbols denote morphological classes: green diamonds globular, red triangles - head-tail, and blue plus signs - other.

to the different cloud definition appeared mostly in highly irregular clouds. That is why we decided to interpret our results using the boundary definition described in Sect. 2. We performed the cloud definition using an automatized procedure and introduced a quantitative morphological description of clouds. According to our sample of the mean values describing parameters, listed in Table 7, star-forming and non-star-forming clouds differ significantly only in size, peak extinction, and mass. Star-forming clouds appear larger than non-star-forming ones and have higher peak extinction and mass. We compared the observed projected axis ratios $b / a$ (see Fig. 5) to the models presented by Jones $\&$ Basu (2002) and estimated the $3 \mathrm{~d}$ shape of our clouds. The distribution found in this study is similar to the modelled distribution obtained using $\xi=0.3$ and $\eta=0.3$ mean intrinsic axis ratios with a $\sigma=0.1$ Gaussian distribution, suggesting that our clouds have near-prolate $3 \mathrm{~d}$ shapes. This is consistent with their formation from large-scale external forcing. 

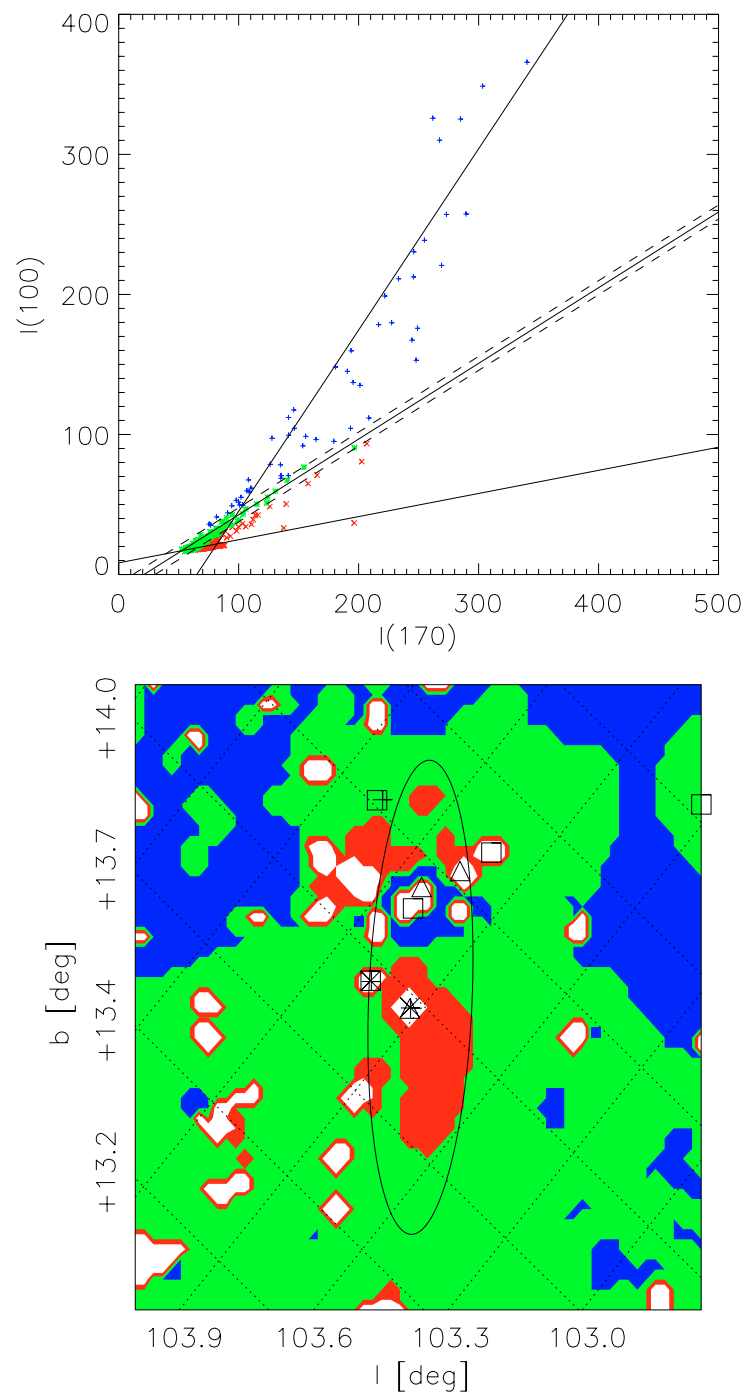

Fig. 11. Top: plot of $I(100)$ versus $I(170)$ for G103.9+14.0. The lines (solid) fitted to the data of the whole region are plotted, as are the colder (red) and warmer (blue) branches, representing temperatures 16.5, 13.3, and $25.1 \mathrm{~K}$ respectively. Bottom: the data were drawn back on the map of the cloud using the same colours. Young point sources of IPSC (crosses) and IFSC (plus signs), T Tauri candidates of 2MASS catalogue (diamonds), ammonia cores (triangles), and $\mathrm{H} \alpha$ stars (squares) are overlaid on the map.

\subsection{FIR colours compared to models}

The obtained FIR colour indices were compared to dust emission models created by Désert et al. (1990). Both observed and modelled data are plotted in Fig. 13. Data points denoted with green and red symbols represent positions observed in non-starforming and star-forming clouds, respectively. Values modelled for dark clouds and cirrus were plotted with black diamonds and triangles, respectively. The dark cloud model data points were computed for values of $A_{\mathrm{V}}$ ranging from $1 \mathrm{mag}$ to $5 \mathrm{mag}$, in 1 mag steps. Values of $I(170) / I(100)$ increase with increasing $A_{\mathrm{V}}$. Cirrus model data points were computed for values of the interstellar radiation field (ISRF), ranging from 0.3 to 1000 times the local ISRF. Values of $I(170) / I(100)$ decrease with increasing ISRF. For both dark clouds and cirrus, the total emission models are denoted with large symbols and the big grain emission models are denoted with small symbols. For globular clouds, the observed data are well-described by the total emission model of
Désert et al. (1990), with a scatter of 1.5. The data points of headtail clouds occupy an area located below $\Delta I(100) / \Delta I(60)=5$ that is conspicuously distinct from that of globular clouds. For regions outside clouds, the model of total emission shows a good match with the upper limit values of $\Delta I(100) / \Delta I(60)$ at each particular $\Delta I(170) / \Delta I(100)$ value.

Since the values of infrared colours characterising the clouds mostly occur in their inner parts, these values are not affected by the cloud definition. The only slight change could be in the fraction of the background contamination; however, it does not affect substantial parts of the plots because the background contamination is negligible in the case of isolated globular clouds, and for head-tail clouds, the background colours can also be distinguished from the cloud colours (see Fig. 13).

\subsection{Mass function}

We constructed the mass function of clouds (see Fig. 14). The power law index $(\alpha=1.70 \pm 0.1)$ found is similar to those obtained by Yonekura et al. (1997) for their close group $\left(\alpha_{\text {Yonekura }}=1.71 \pm 0.26\right)$. The obtained mass function is:

$\mathrm{d} N_{\text {cloud }} / \mathrm{d} M_{\text {cloud }}=6.1 \times 10^{2}\left(M_{\text {cloud }} / M_{\odot}\right)^{-1.70} M_{\odot}^{-1}$.

Like Yonekura et al. (1997), we fitted the power law above $20 M_{\odot}$. Since the sample is complete above $11.4 M_{\odot}$ (see Table 4), the derived cloud-mass function index is free of bias, due to an incomplete low-mass cloud sample, even for the most distant layer. We checked the mass function for each distance group of clouds separately and found the same $\alpha$ index within the obtained error. Thus, the given value is also free of the bias of different distances.

In addition, we checked the effect of using different fixed extinction levels of cloud boundary definition, in the $\log M$ range between $1.6 \mathrm{mag}$ and $2.8 \mathrm{mag}$ in $0.2 \mathrm{mag}$ steps, and found that, the obtained values of $\alpha$ vary around the mean of 1.81 with a 0.11 scatter, which does not conflict with our previous result. Other authors obtained similar results for different cloud samples. Tachihara et al. (2002) found values of 2.6 and 1.5 for observed $\mathrm{C}^{18} \mathrm{O}$ cores in mass ranges above and below $\log M=$ 1.75 , while slopes obtained by Klessen (2001), based on different theoretical models of clump mass function, were 1.5 and 2.

\subsection{Cloud-complex mass ratio}

The mean ratio of the total cloud mass in a complex to the total mass of the complex was found to be $21 \%$ for the whole region. Table 5 shows the values for all of the individual complexes. Cep SW, apparently related to the most prominent void in the region (\#2), has a relatively high ratio. In contrast, Cep SE, which also appears to be connected to void\#2, does not have such a high ratio. This may be caused by the confusion of a part of Cep $\mathrm{N}$ that may overlap Cep SE, and which also has a low mass ratio value. The relatively low value obtained for Cep NW is due to the rareness of high extinction in the complex, in addition to the small number of clouds. The low value obtained for Cep S may be affected by the fact that this complex is situated at a low galactic latitude, hence the value may be influenced by the galactic plane.

\subsection{Star-forming efficiency}

There is a total mass $3.1 \times 10^{4} M_{\odot}$ in the clouds and 98 YSO candidates in the $\mathrm{H} \alpha$ surveys, done by Kun (1998), 2MASS 

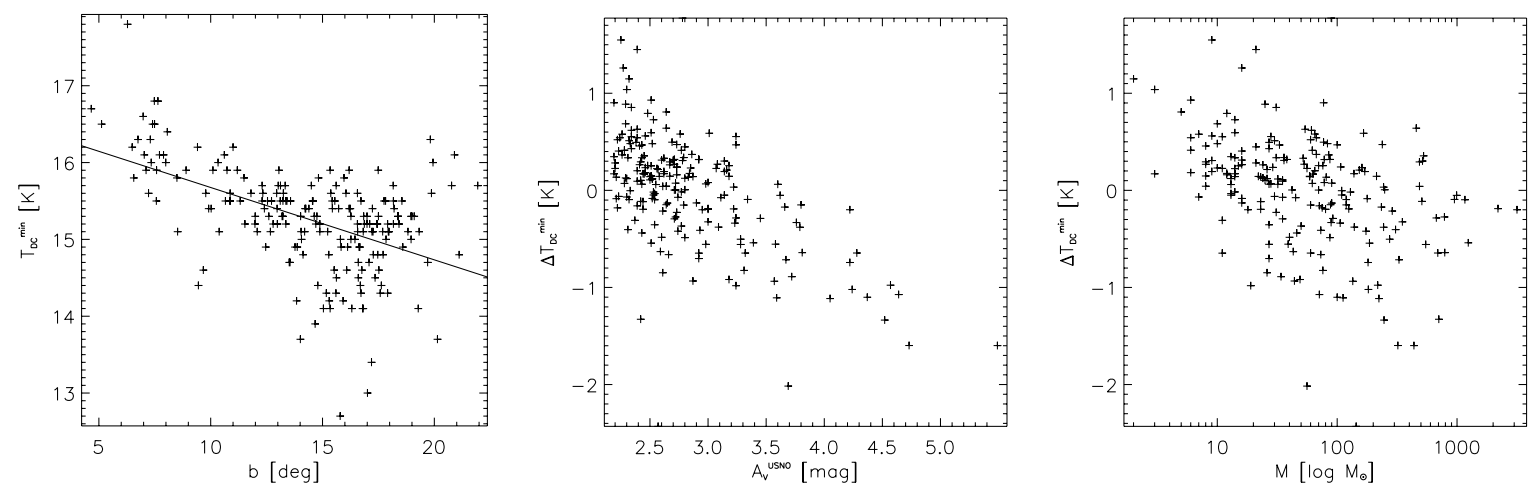

Fig. 12. Change of $T_{\mathrm{DC}}^{\min }$ with the galactic latitude (left), the relation between $\Delta T_{\mathrm{DC}}^{\min }$ and optical extinction (middle), and the relation between $\Delta T_{\mathrm{DC}}^{\min }$ and the masses of clouds (right).
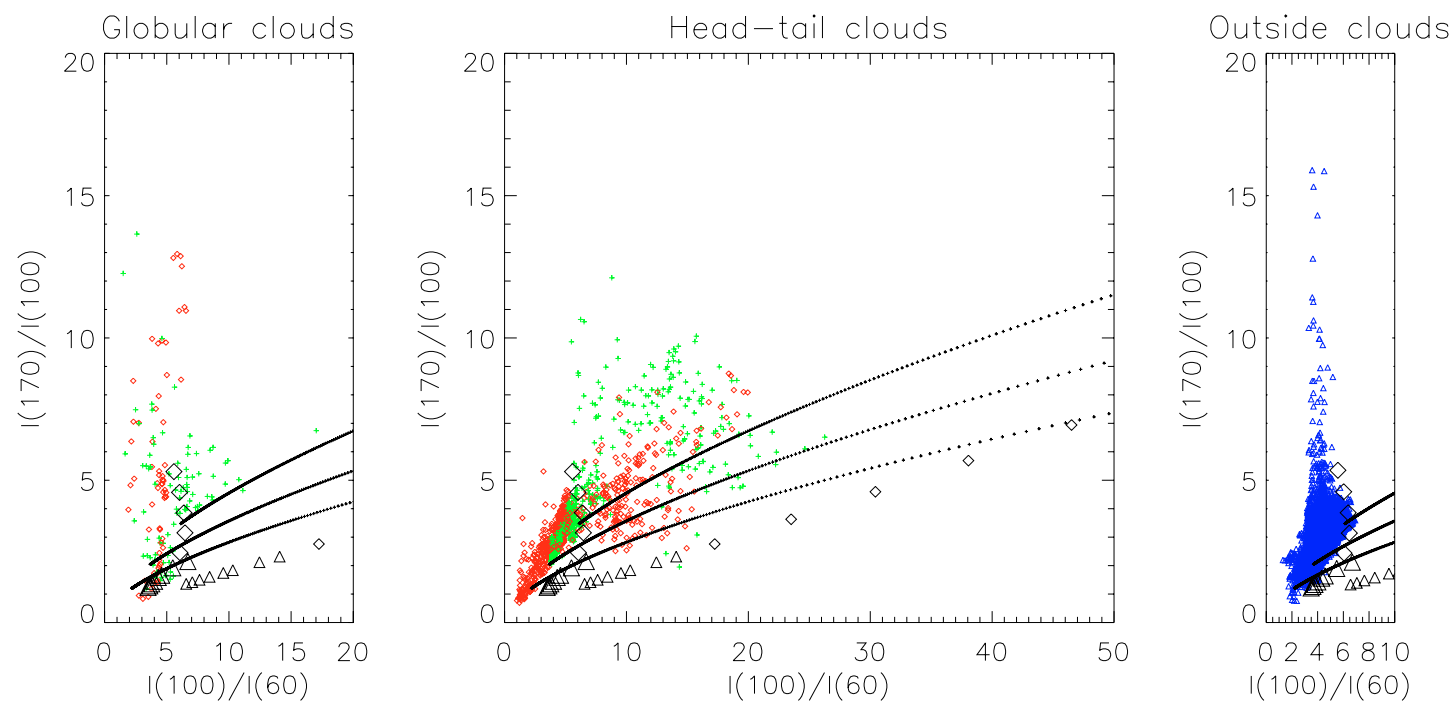

Fig. 13. Two-colour plot for positions inside globular (left) and head-tail (middle) clouds. Red symbols belong to clouds with point sources, green ones to clouds without point sources. Greybody estimations were plotted with $-1,-2$, and -3 emissivity (upwards). Small and large symbols represent models for big grain and total emission, respectively. Diamonds indicate points of the dark cloud model at $A_{\mathrm{V}}=1.0,2.0,3.0,4.0$, and 5.0 towards increasing $I(170) / I(100)$ values. Triangles are points of cirrus model at ISRF $0.3,0.5,2.0,3.0,5.0,10,100$, and $1000 \times$ LISRF towards decreasing $I(170) / I(100)$ values. Data for points outside clouds are also plotted (right).

Table 7. Mean values of morphological parameters for all clouds. The values are noted separately for clouds with and without YSO candidates.

\begin{tabular}{lrrrrrrrr}
\hline \hline & $a$ & $b / a$ & $d p / a$ & sep & sym & irr & $A_{\mathrm{V}}$ & $\log M$ \\
\hline All & $23.9 \pm 16.7$ & $0.51 \pm 0.18$ & $0.21 \pm 0.12$ & $0.29 \pm 0.11$ & $0.70 \pm 0.17$ & $0.42 \pm 0.18$ & $2.8 \pm 0.6$ & $1.71 \pm 0.61$ \\
\hline SF & $36.5 \pm 24.5$ & $0.48 \pm 0.16$ & $0.21 \pm 0.14$ & $0.32 \pm 0.12$ & $0.70 \pm 0.16$ & $0.44 \pm 0.23$ & $3.3 \pm 0.7$ & $2.18 \pm 0.61$ \\
NSF & $21.3 \pm 13.2$ & $0.51 \pm 0.18$ & $0.21 \pm 0.12$ & $0.28 \pm 0.11$ & $0.70 \pm 0.17$ & $0.41 \pm 0.17$ & $2.7 \pm 0.5$ & $1.61 \pm 0.56$ \\
\hline
\end{tabular}

(Cutri et al. 2003), IPSC (Beichman et al. 1988), and IFSC (Moshir et al. 1990), associated with clouds. Assuming $0.5 M_{\odot}$ for each YSO candidate, we obtain an average star-forming efficiency of $0.16 \%$ in the clouds.

Only 36 clouds (17\%) are associated with YSO candidates: 4 of the 12 head-tail clouds $(33 \%)$ and 3 of the 12 globular clouds $(25 \%)$. Star-forming efficiencies for globular and headtail clouds are $0.52 \%$ and $0.56 \%$, respectively, which is relatively high with respect to the average of the whole cloud sample $(0.16 \%)$.

The effect of our choice of cloud definition has not proved to be critical in this aspect. We checked the results of the constant $A_{\mathrm{V}}^{\mathrm{USNO}}=2.0 \mathrm{mag}$ boundary definition. For most of the headtail clouds, the two definitions do not differ significantly, and the total mass does not either. The number of YSOs found in these clouds is the same with both definitions. Therefore, the star-forming efficiency of clouds in this group does not change significantly. In the case of globular clouds, the total mass decreased significantly because of the notably smaller cloud sizes that were the results of the constant boundary level. However, the number of YSOs found in them remained the same. Therefore, the star-forming efficiency obtained was even higher. For the other clouds, the constant boundary level also resulted in somewhat smaller cloud sizes, however the number of YSOs in these clouds decreased as well, and thus their mean star-forming efficiency increased from $0.13 \%$ to $0.16 \%$. This confirms that our choice of cloud definition does not significantly affect our results.

Star-forming efficiency for each individual complex is also given in Table 5. Cep S and Cep NE show higher star-forming 


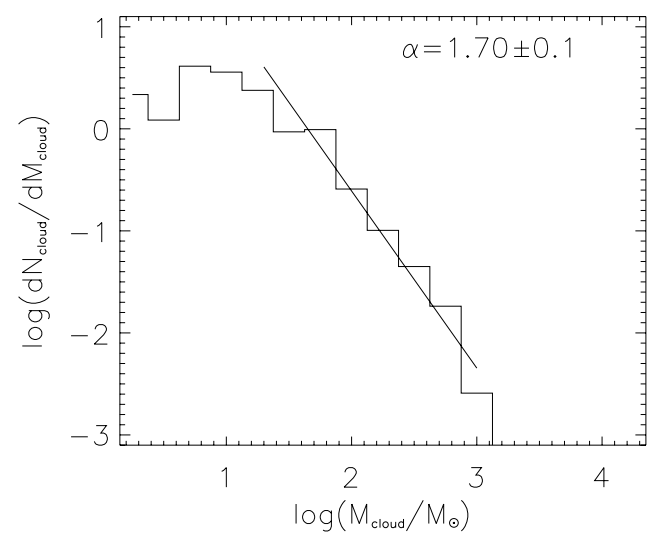

Fig. 14. Cloud mass function and the fitted power law index: $\mathrm{d} N_{\text {cloud }} / \mathrm{d} M_{\text {cloud }}=6.1 \times 10^{2}\left(M_{\text {cloud }} / M_{\odot}\right)^{-1.70} M_{\odot}^{-1}$.
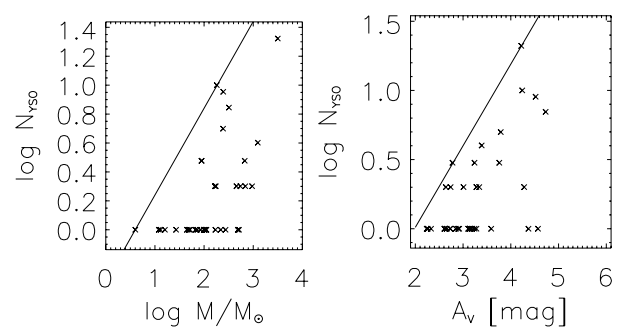

Fig. 15. Number of YSO candidates per cloud versus cloud mass and peak extinction.

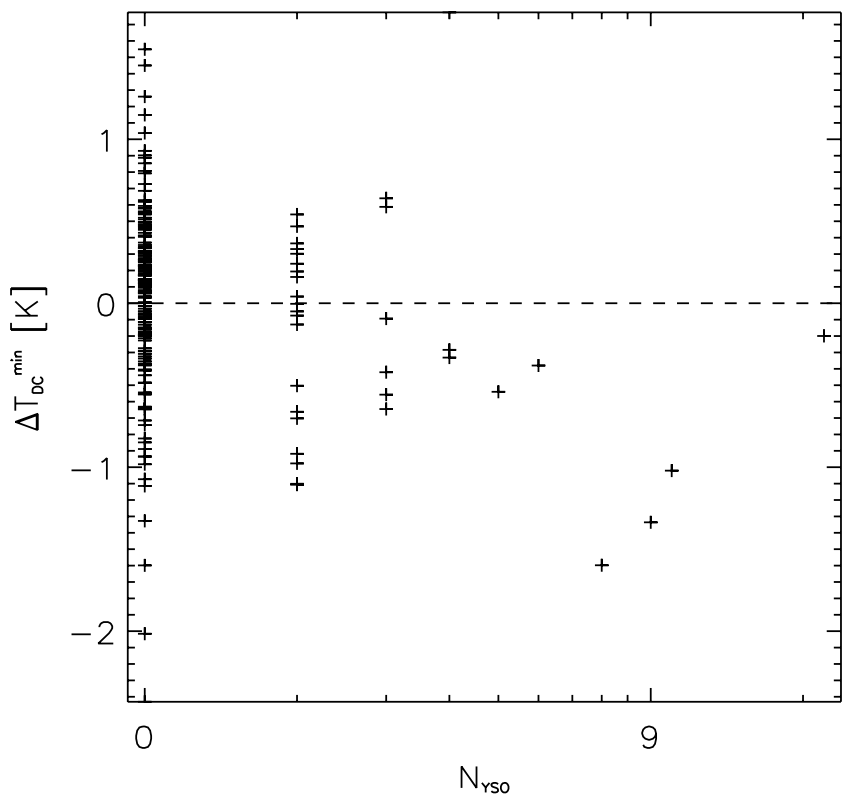

Fig. 16. Relation of $\Delta T_{\mathrm{DC}}^{\mathrm{min}}$ to the number of YSO candidates.

efficiency than the average, Cep $\mathrm{N}$ and Cep SE show notably low star-forming efficiency, while Cep NW does not show star formation at all. Although Cep SE does not show particularly high star-forming efficiency on average (see Fig. 1), the southern and eastern edges of the complex show notably higher efficiency than its central parts do. The locations of the active regions correspond to the arc-like area showing $12 \mu \mathrm{m}$ excess in Fig. 9. A relative increase of $I(12)$ may refer to an increase in the radiation of small dust particles. That is either due to an increase of their abundance or of their excitation. Both may occur in bubble shells (Kiss et al. 2004). The structure seen as $12 \mu \mathrm{m}$ excess is extended from the eastern edge of Cep SE along the complex to the eastern part of Cep SW, which also shows star-forming activity. In addition, Cep $\mathrm{S}$ shows both $12 \mu \mathrm{m}$ excess and star-forming activity. All these three complexes appear to be connected to void \#2, which coincides with the FIR loop GIRL G109+11 (Kiss et al. 2004). The observed features around void \#2 probably originate in the relatively warm and dense material compressed by the shell that is also seen in the extinction pattern. This shell appears to play an important role in generating higher star-forming activity. This hypothesis is in agreement with results determined by Lee et al. (2005), who found the star formation to take place in the outer layers of bright-rimmed clouds of Orion, suggesting triggered star formation.

In agreement with our results, Tachihara et al. (2002) found that the formation of stellar clusters tends to associate with head-tail structured clouds, suggesting the importance of external influence. For small globules, the influence of nearby earlytype stars on star formation was pointed out by Froebrich et al. (2005).

We found an upper limit for star-forming efficiency of clouds as a function of cloud mass, $N_{\text {YSO }}<0.44 \times M^{0.60}$ (see Fig. 15), and a similar relation between star-forming efficiency and cloud peak extinction, $\log N_{\mathrm{YSO}}<0.59 \times A_{\mathrm{V}}^{\mathrm{USNO}}-1.17$.

The relation of latitude corrected $\Delta T_{\min }$ and the number of young star-like sources in the clouds were also plotted (Fig. 16). The mean of the corrected temperatures of clouds without such YSO candidates is somewhat higher $\left(\overline{\Delta T_{\min }}>0\right)$, while clouds with YSO candidates have lower corrected temperatures $\left(\overline{\Delta T_{\min }}<0\right)$, on average. To test the validity of the results obtained with our choice of cloud definition, we checked the effects of an obvious alternative way: definition using constant contour level. We found that though the numerical results obtained would change slightly, this would not affect the validity of our conclusions.

\section{Summary}

i) We presented the $A_{\mathrm{V}}$ extinction map of $\mathrm{a} \approx 256$ square degree area of the Cepheus region.

ii) 8 cloud complexes and 4 voids were identified, and 208 dark clouds were localised using an automated procedure. 86 of these dark clouds have no previously catalogued associated cloud.

iii) We defined quantitative morphological parameters and searched for clouds of certain special morphological types, in particular, globular and head-tail shapes.

iv) The observed distribution of cloud axis ratio has been found to agree with that of modelled near-prolate ellipsoidal clouds with random alignment.

$v$ ) We found a relation between dust colour temperature and galactic latitude in the region: $T_{\mathrm{DC}}=19.8[\mathrm{~K}]-$ $0.30[\mathrm{~K} / \mathrm{deg}] \times b$.

vi) FIR colour indices of dark clouds and the inter-cloud medium were found to be different, as were the colours of clouds with different morphologies.

vii) We found relations between minimum temperatures of clouds and their optical and NIR extinction: more opaque clouds tend to be colder.

viii) We also found that the number of YSO candidates in clouds increases with increasing extinction and decreasing temperature. 
ix) The mass spectrum of clouds was derived, and the power law index was found to be $\alpha=1.70 \pm 0.1$, in good agreement with former results obtained from $\mathrm{CO}$ observations.

$x)$ We pointed out the signs of connection between GIRL G109+11 and the active, triggered star formation of adjacent cloud complexes.

xi) We showed that star formation is more effective in globular and head-tail clouds than in other clouds and that its efficiency mainly depends on cloud mass and peak extinction.

xii) We derived empirical formulae to estimate the upper limits of star-forming efficiency from cloud mass and peak extinction.

Acknowledgements. We would like to thank Lajos Balázs for supplying his FORTRAN program of the Wainscoat model and Péter Ábrahám for the DIRBE ZSMA data. We thank László Szabados for his critical reading of the manuscript. We are grateful to B.G. Elmegreen and K. Mattila for their inspiration and useful comments. This research has made use of the USNOFS Image and Catalogue Archive operated by the United States Naval Observatory, Flagstaff Station (http://www.nofs.navy.mil/data/fchpix/). This publication makes use of data products from the Two Micron All Sky Survey, which is a joint project of the University of Massachusetts and the Infrared Processing and Analysis Center, funded by the National Aeronautics and Space Administration and the National Science Foundation. This research has made use of the NASA/IPAC Infrared Science Archive, which is operated by the Jet Propulsion Laboratory, California Institute of Technology, under contract with the National Aeronautics and Space Administration. The ISOPHOT Data Centre at MPIA is supported by Deutsches Zentrum für Luft und Raumfahrt (DLR) with funds of the Bundesministerium für Bildung und Forschung, grant No. 50QI0201. This work was partly supported by the OTKA grants \#T-043773, \#T-034584, and \#T-049082.

\section{References}

Allen, C. V. 1973, Astrophysical Quantities (London: Athlone)

Balázs, L. G., Ábrahám, P., Kun, M., Kelemen, J., \& Tóth, L. V. 2004, A\&A, 425,133

Barnard, E. E. 1927, A Photographic Atlas of Selected Regions of the Milky Way, ed. E. B. Frost, \& M. R. Calvert (Washington: Carnegie Institution of Washington)

Beichman, C. A., Myers, P. C., Emerson, J. P., et al. 1986, ApJ, 307, 337

Beichman C., Neugebauer, G., Habing, H. J., Clegg, P. E., \& Chester, T. J., ed. 1988, Infrared astronomical satellite (IRAS) Catalogs and Atlases, Vol. 1, Explanatory Supplement

Bogun, S., Lemke, D., Klaas, U., et al. 1996, A\&A, 315, L71

Benson, P. J., \& Myers, P. C. 1989, ApJS, 71, 89

Burton, W. B., \& Hartmann, D. 1994, ApJS, 217, 189

Cambrésy, L. 1999a, A\&A, 345, 965
Cambrésy, L. 1999b, in Proceedings of Star Formation 1999, held in Nagoya, Japan, ed. T. Nakamoto (Nobeyama Radio Observatory), 90

Cambrésy, L., Beichman, C. A., Jarrett, T. H., \& Cutri, R. M. 2002, AJ, 123, 2559

Clemens, D. P., \& Barvainis, R. 1988, ApJS, 68, 257

Cutri, R. M., Skrutskie, M. F., van Dyk, S., et al. 2003, VizieR Online Data Catalog, 2246

Désert, F.-X., Boulanger, F., \& Puget, J. L. 1990, A\&A, 237, 215

Dickman, R. L. 1978, AJ, 83, 363

Dobashi, K., Uehara, H., Kandori, R., et al. 2005, PASJ, 57S, 1

Evans, N. J. 1999, ARA\&Ap, 37, 311

Froebrich, D., Scholz, A., Eislöffel, J., \& Murphy, G. C. 2005, A\&A, 432, 575

Harjunpää, P., Liljeström, T., \& Mattila, K. 1991, A\&A, 249, 493

Hauser, M. G., Kelsall, T., Leisawitz, D., \& Weiland, J. 1997, COBE Diffuse Infrared Background Experiment (DIRBE) Explanatory Supplement (COBE Science Working Group)

Hubble, E. 1934, ApJ, 79, 8

Jenkins, E. B., \& Savage, B. D. 1974, ApJ, 187, 243

Jones, C. E., \& Basu, S. 2002, ApJ, 569, 280

Kiss, Cs., Tóth, L. V., Moór, A., et al. 2000, A\&A, 363, 755

Kiss, Cs., Moór, A., \& Tóth, L. V. 2004, A\&A, 418, 131

Kiss, Z., Tóth, L. V., Miller, M., \& Yonekura, Y. 2004, Balt. Astr., 13, 430

Klessen, R. S. 2001, ApJ, 556, 837

Krause, O. 2003, Ph.D. Thesis, University of Heidelberg

Kun, M. 1998, ApJS, 115, 59

Lebrun, F. 1986, ApJ, 306, 16

Lee, H. T., Chen, W. P., Zhang, Z. W., \& Hu, J. Y. 2005, ApJ, 624, 808

Lemke, D., Klaas, U., Abolins, J., et al. 1996, A\&A, 315, 64

Lombardi, M., \& Alves, J. 2001, A\&A, 377, 1023

Lynds, B. T. 1962, ApJS, 7, 1

Meyer, R. M., Calvet, N., \& Hillenbrand, L. A. 1997, AJ, 114, 288

Monet, D., Bird, A., Canzian, B., et al. 1998, VizieR Online Data Catalog, 1252

Moshir, M., Copan, G., Conrow, T., et al. 1990, Iras Catalogs, The Faint Source Catalog, Version 2.0

Müller, T. G., Hotzel, S., \& Stickel, M. 2002, A\&A, 389, 665

Paturel, G., Petit C., Prugniel, P., et al. 2003, A\&A, 412, 45

Racine, R. 1968, AJ, 73, 233

Stickel, M., Bogun, S., Lemke, D., et al. 1998, A\&A, 336, 116

Straižys, V., Černis, K., Kazlauskas, A., \& Meištas, E. 1992, BAAS, 1, 149

Tachihara, K., Onishi, T., Mizuno, A., \& Fukui, Y. 2002, A\&A, 385, 909

Taylor, D. K., Dickman, R. L., \& Scoville, N. Z. 1987, ApJ, 315, 104

Tóth, L. V., Hotzel, S., Krause, O., et al. 2000, A\&A, 364, 769

Tóth, L. V., \& Horváth, A. 1996, in The Role of Dust in the Formation of Stars, ed. H. U. Käufl, \& R. Siebenmorgen (Springer), 129

Wainscoat, R. J., Cohen, M., Wolk, K., Walker, H. J., \& Schwartz, D. E. 1992, ApJS, 83, 111

Ward-Thompson, D. 2002, Science, 295, 76

Wheelock, S., Gautier III, T. N., Chillemi, J., et al. 1991, BAAS, 23, 908

Yonekura, Y., Dobashi, K., Mizuno, A., Ogawa, H., \& Fukui, Y. 1997, ApJS, 110,21 